\title{
PERVERSE COHERENT SHEAVES ON BLOW-UPS AT CODIMENSION 2 LOCI
}

\author{
NAOKI KOSEKI
}

\begin{abstract}
Let $f: X \rightarrow Y$ be the blow-up of a smooth projective variety $Y$ along its codimension two smooth closed subvariety. In this paper, we show that the moduli space of stable sheaves on $X$ and $Y$ are connected by a sequence of flip-like diagrams. The result is a higher dimensional generalization of the result of Nakajima and Yoshioka, which is the case of $\operatorname{dim} Y=2$. As an application of our general result, we study the birational geometry of the Hilbert scheme of two points.
\end{abstract}

\section{INTRODUCTION}

1.1. Main result. In this paper, we consider the following natural question:

Question 1.1. Let $X \rightarrow Y$ be a birational map between varieties. What is the relation between the moduli space of stable sheaves on $Y$ and that of $X$ ?

There are several works answering Question 1.1 in various situations (1, 2, 11, 12, 13, 14, 18, 19]). In particular, Nakajima and Yoshioka proved the following theorem:

Theorem $1.2([13)$. Let $(Y, H)$ be a polarized smooth projective surface, $f: X \rightarrow$ $Y$ the blow-up at a point. Let $v=\left(v_{0}, v_{1}, v_{2}\right) \in H^{2 *}(X ; \mathbb{Q})$ be the Chern character of a sheaf with $v_{0}>0, \operatorname{gcd}\left(v_{0}, f^{*} H \cdot v_{1}\right)=1$.

Then there exists a diagram of projective schemes (1.1)

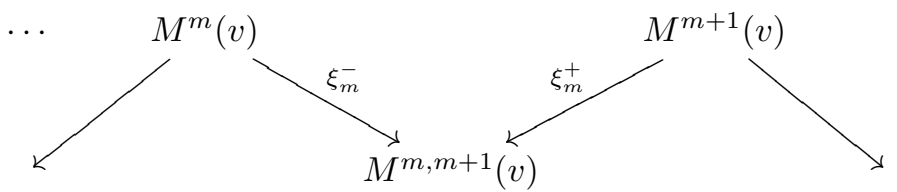

such that

(1) For an integer $m \in \mathbb{Z}_{\geq 0}$, the scheme $M^{m}(v)$ is the moduli space of $m$ stable sheaves with Chern character $v$, and $M^{m, m+1}(v)$ is a scheme whose closed points corresponds to $m$-stable and $(m+1)$-stable sheaves with various Chern characters (see Definition 2.7 for the notion of m-stability).

(2) If there exists an element $w \in H^{2 *}(Y ; \mathbb{Q})$ with $v=f^{*} w$, then $M^{0}(v)$ is isomorphic to the moduli space of stable sheaves on $Y$.

(3) For every sufficiently large $m, M^{m}(v)$ is isomorphic to the moduli space of stable sheaves on $X$.

(4) For every integer $m \in \mathbb{Z}_{\geq 0}$, the fiber over $\xi_{m}^{ \pm}$is the Grassmann variety.

In the above theorem, the notion of perverse coherent sheaves plays an important role. In particular, it leads us to define natural stability conditions indexed by $m \in \mathbb{Z}_{\geq 0}$, called $m$-stablilty. The $m$-stability is similar to the Gieseker stability. However, an $m$-stable sheaf may have a torsion subsheaf supported on the $f$-exceptional curve. Such a generalization of stability enables us to connect the moduli space of stable sheaves on $X$ and that of $Y$. 
The notion of perverse coherent sheaves was introduced by Bridgeland (cf. [2]). A perverse coherent sheaf is an element of the heart of a certain bounded t-structure (called perverse heart and denoted by $\operatorname{Per}(X / Y)$ ) in the derived category of coherent sheaves on $X$. The heart $\operatorname{Per}(X / Y)$ encodes the information of the morphism $f$ and it can be defined more general situation. In particular, if we have the blow-up $f: X \rightarrow Y$ of a smooth projective variety along its codimension two smooth closed subvariety, we can define the perverse heart $\operatorname{Per}(X / Y) \subset D^{b}(X)$. In this setting, we generalize the result of the paper [13. The precise statement of our main theorem of the present paper is the following:

Theorem 1.3. Let $(Y, H)$ be a polarized smooth projective variety of dimension $n \geq 2, f: X \rightarrow Y$ the blow-up along its codimension two smooth closed subvariety. Let $v=\left(v_{0}, v_{1}, \cdots\right) \in H^{*}(X ; \mathbb{Q})$ be a Chern character with $v_{0}>0$, $\operatorname{gcd}\left(v_{0}, f^{*} H^{n-1} \cdot v_{1}\right)=1$. Then there exists a diagram of projective schemes as in (1.1) such that

(1) The scheme $M^{m}(v)$ is the moduli space of $m$-stable sheaves with Chern character $v$, and $M^{m, m+1}(v)$ is a scheme whose closed points corresponds to $m$-stable and $(m+1)$-stable sheaves with various Chern characters $(m \in$ $\mathbb{Z}_{\geq 0}$ ).

(2) If there exists an element $w \in H^{2 *}(Y ; \mathbb{Q})$ with $v=f^{*} w$, then $M^{0}(v)$ is isomorphic to the moduli space of stable sheaves on $Y$.

(3)' For every sufficiently large $m$, the moduli space of stable sheaves on $X$ is embedded into $M^{m}(v)$ as an open and closed subscheme.

(4)' For every integer $m \in \mathbb{Z}_{\geq 0}$, the fiber over $\xi_{m}^{ \pm}$is the certain Quot scheme.

The above theorem is a summary of Corollary 4.2 Proposition 4.4, Proposition 5.5. and Proposition 5.6. See the next subsection for the summary of differences between Theorem 1.2 and Theorem 1.3 .

Using Theorem 1.3, we also study the birational geometry of Hilbert scheme of two points:

Theorem 1.4 (Corollary 6.12, Collollary 6.16). Let $f: X \rightarrow Y$ be as in Theorem 1.3. let $v:=(1,0, \cdots, 0,-2) \in H^{2 *}(X ; \mathbb{Q})$. Assume that $H^{1}\left(\mathcal{O}_{Y}\right)=0$. Then we have a diagram of projective varieties

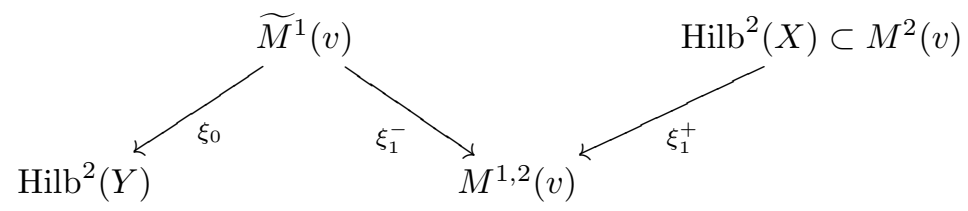

such that

(1) When $\operatorname{dim} Y=2$ (resp. 3, $\geq 4$ ), $\operatorname{Hilb}^{2}(X) \rightarrow \widetilde{M}^{1}(v)$ is a flip (resp. a flop, an anti-flip).

(2) The morphism $\xi_{0}$ is the contraction of a $K$-negative extremal ray.

Moreover, we will determine all the fibers over $\xi_{0}$. When $\operatorname{dim} Y \geq 3$, we see that some fibers of $\xi_{0}$ are not the Grassmann varieties (see Lemma 6.14). This is the new phenomenon which does not happen in dimension 2 (see Theorem 1.2 (4)).

1.2. Difference between Theorem $\mathbf{1 . 2}$ and Theorem 1.3. The idea of the proof of our main theorem is similar to that of [13. However, we need to modify the proofs in various points, which are not so straightforward. Let us explain about the differences. Let $f: X \rightarrow Y$ be as in Theorem [1.3. $D \subset X$ the $f$-exceptional divisor. 
0-stability and 1-stability. One of the key part of the argument is to describe the difference between 0 -stability and 1-stability. Here, a coherent sheaf $E$ is said to be 0 -stable if $E$ is a perverse coherent sheaf and $f_{*} E$ is a slope stable torsion free sheaf on $Y$. On the other hand, we say that $E$ is 1 -stable if $E(-D)$ is 0 -stable. To explain the argument, let $E^{-} \in \operatorname{Coh}(X)$ be a 0 -stable sheaf. In the surface cace, Nakajima and Yoshioka proved that the obstruction for the 1-stability is captured by looking at the vector space $V:=\operatorname{Hom}\left(\mathcal{O}_{D}(-1), E^{-}\right)$. In fact, we can show that the evaluation morphism $e v: V \otimes \mathcal{O}_{D}(-1) \rightarrow E$ is always injective and hence we have a short exact sequence

$$
0 \rightarrow V \otimes \mathcal{O}_{D}(-1) \rightarrow E^{-} \rightarrow F:=\operatorname{Coker}(e v) \rightarrow 0
$$

in $\operatorname{Coh}(X)$. Furthermore, we can also show that $F$ is 1-stable. Similarly, we can construct a 0 -stable sheaf from a 1 -stable sheaf $E^{+}$by looking at the vector space $\operatorname{Hom}\left(E^{+}, \mathcal{O}_{D}(-1)\right)$. In this way, we get a (set-theoretical) diagram (1.1) by sending $E^{-} \in M^{0}(v)$ to $F \in M^{0,1}(v)$, etc.

However, in higher dimension, not only the sheaf $\mathcal{O}_{D}(D)$, but also various subsheaves of $E^{-}$become the obstruction for 1-stability. Hence we should take the maximum subsheaf among them. We take such a subsheaf by using torsion pairs on $\operatorname{Coh}(X)$. See Definition 3.1 for the definition of torsion pairs. Assume that we have a torsion pair on $\operatorname{Coh}(X)$. Then we have the canonical decomposition

$$
0 \rightarrow T \rightarrow E^{-} \rightarrow F \rightarrow 0
$$

with respect to the torsion pair. Using such a decomposition, we will see that the difference between 0-stability and 1-stability is captured by certain torsion pairs on $\operatorname{Coh}(X)$, which are defined in Definition 3.3 and Definition 3.4. In the surface case, we can easily see that the exact sequence (1.2) is nothing but the decomposition with respect to our torsion pair.

Scheme structure on $M^{0,1}(v)$. The another key point is how to define the scheme structure on $M^{0,1}(v)$. As a set, $M^{0,1}(v)$ is the disjoint union of the moduli spaces of 0 -stable and 1-stable sheaves with various Chern characters. In the surface case, Nakajima and Yoshioka used the moduli space of perverse coherent systems to define the scheme structure on $M^{0,1}(v)$.

Instead, we use the natural morphisms between moduli spaces. More precisely, we will show that the set-theoretical diagram is naturally identified with the diagram

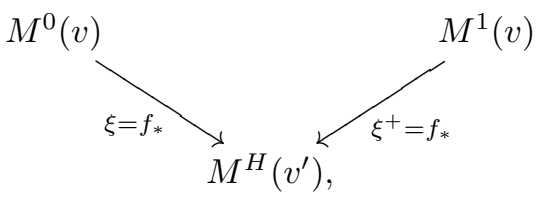

where $M^{H}\left(v^{\prime}\right)$ denotes the moduli space of Gieseker stable sheaves on $Y$. Hence we define a scheme structure on $M^{0,1}(v)$ as the union of the scheme-theoretic images of $\xi$ and $\xi^{+}$. To be more precise, let $I, I^{+} \subset \mathcal{O}_{M^{H}\left(v^{\prime}\right)}$ be ideal sheaves defining the scheme theoretic images of $\xi, \xi^{+}$, respectively. Then the scheme $M^{0,1}(v)$ is defined to be a closed subscheme of $M^{H}\left(v^{\prime}\right)$ whose defining ideal is $I \cap I^{+}$. Note that Nakajima and Yoshioka do the essentially same thing and our approach is inspired by them.

Moduli space of stable sheaves on $X$. We mention about the difference between Theorem 1.2 (3) and Theorem 1.3 (3)'. According to (3)' in Theorem 1.3, we have an open and closed embedding from the moduli space of stable sheaves on $X$ to the moduli space of $m$-stable sheaves with sufficiently large integer $m \in \mathbb{Z}_{\geq 0}$. In the surface case, Nakajima and Yoshioka showed that the above embedding is actually an isomorphism. To show that, they used the following speciality of the surface: Let 
$E$ be a torsion free sheaf on a surface. Then the quotient $E^{D D} / E$ is 0-dimensional, where $E^{D D}$ is the double dual of $E$. In particular, we have $\chi\left(E^{D D} / E\right) \geq 0$. In the higher dimension case, we do not have such a positivity of the quotient sheaf $E^{D D} / E$, which is crucial in the proof given by Nakajima and Yoshioka. In the present paper, we only prove that when $n=3$ and $v=(1,0, \cdots, 0,-k)$, the embedding given in Theorem 1.3 (3)' is actually an isomorphism (see Example 4.6).

1.3. Plan of the paper. The paper is organized as follows. In Section 2, we collect the notions and the properties about perverse coherent sheaves on blow-ups. In Section 3, we describe the diagram (1.1) set-theoretically. In the proofs, we will use certain torsion pairs. In Section 4, we explain the relationship between the $m$-stability and the Gieseker stability on both blow-up and blow-down varieties. In Section 5, we realize the diagram (1.1) scheme-theoretically. In Section 6, we study the diagram (1.1) more explicitly in the case of Hilbert scheme of two points.

Notation and Convention. In this paper, we always work over the complex number field $\mathbb{C}$. We use the following notanions:

- For a variety $X$, we denote by $\operatorname{Coh}(X)$ the Abelian category of coherent sheaves on $X$.

- For a variety $X$, we denote by $D^{b}(X):=D^{b}(\operatorname{Coh}(X))$ the bounded derived category of coherent sheaves on $X$.

- For a set of objects $S \subset D^{b}(X)$, we denote by $\langle S\rangle$ the extension closure of $S$ in $D^{b}(X)$.

- For a proper morphism $f: M \rightarrow N$ between varieties and $E, F \in \operatorname{Coh}(M)$, we denote by $\mathcal{E} x t_{f}^{q}(E, F)$ the $q$-th derived functor of $f_{*} \mathcal{H o m}(E, F)$.

- For a variety $X$ and $E, F \in \operatorname{Coh}(X)$, we define $\operatorname{hom}(E, F):=\operatorname{dim} \operatorname{Hom}(E, F)$ and $\operatorname{ext}^{i}(E, F):=\operatorname{dim} \operatorname{Ext}^{i}(E, F)$.

\section{Perverse COHEREnt sheaves AND their moduli SPACES}

Throughout the paper, we use the following notations: Let $Y$ be a smooth projective variety, $C \subset Y$ a codimension 2 smooth closed subvariety of $Y$. Let $X:=\mathrm{Bl}_{C} Y$ be the blow-up of $Y$ along $C, D \subset X$ the exceptional divisor. Hence we have the following diagram:

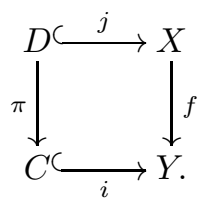

2.1. Perverse coherent sheaves on blow-ups. In this subsection, we collect the results about the perverse coherent sheaves. First note that the following two conditions hold:

(1) $\mathbf{R} f_{*} \mathcal{O}_{X} \cong \mathcal{O}_{Y}$,

(2) $\operatorname{dim} f^{-1}(y) \leq 1$ for all $y \in Y$.

In such a situation, Bridgeland introduced the heart of a bounded t-structure $\operatorname{Per}(X / Y)$ on $D^{b}(X)$ (called perverse heart) as follows (cf. [2, 20]):

$$
\operatorname{Per}(X / Y):=\left\{\begin{array}{ll} 
& f_{*} \mathcal{H}^{-1}(E)=0 \\
E \in D^{b}(X):: & \mathbf{R}^{1} f_{*} \mathcal{H}^{0}(E)=0, \operatorname{Hom}\left(\mathcal{H}^{0}(E), \mathcal{C}^{0}\right)=0 \\
& \mathcal{H}^{i}(E)=0, \quad i \neq-1,0
\end{array}\right\}
$$


where $\mathcal{C}:=\left\{E \in D^{b}(X): \mathbf{R} f_{*} E=0\right\}$ and $\mathcal{C}^{0}:=\mathcal{C} \cap \operatorname{Coh}(X)$. In 2, the heart $\operatorname{Per}(X / Y)$ is denoted as ${ }^{-1} \operatorname{Per}(X / Y)$. We call an element of $\operatorname{Per}(X / Y) \cap \operatorname{Coh}(X)$ as a perverse coherent sheaf.

We will use the following lemma:

Lemma 2.1. We have equalities

$$
\mathcal{C}^{0}=\pi^{*} \operatorname{Coh}(C) \otimes \mathcal{O}(D)=f^{*} \operatorname{Coh}(C) \otimes \mathcal{O}(D) .
$$

Proof. The equality $\pi^{*} \operatorname{Coh}(C) \otimes \mathcal{O}(D)=f^{*} \operatorname{Coh}(C) \otimes \mathcal{O}(D)$ follows from the isomorphism of functors

$$
f^{*} i_{*} \cong j_{*} \pi^{*}: \operatorname{Coh}(C) \rightarrow \operatorname{Coh}(X) .
$$

To prove the first equality, first note that we have $\mathcal{C}=\pi^{*} D^{b}(C) \otimes \mathcal{O}(D)$ by the following semi-orthogonal decomposition (cf. [15])

$$
D^{b}(X)=\left\langle\pi^{*} D^{b}(C) \otimes \mathcal{O}(D), \mathbf{L} f^{*} D^{b}(Y)\right\rangle .
$$

Furthermore, by the exactness of the functors $\pi^{*}$ and $(-) \otimes \mathcal{O}(D)$, we have the inclusion

$$
\mathcal{C}^{0} \subset \pi^{*} \operatorname{Coh}(C) \otimes \mathcal{O}(D) .
$$

Since both of them are hearts of bounded t-structures on $\mathcal{C}$, they must coincide.

The following result is due to Van den Bergh:

Theorem 2.2 (20, Proposition 3.3.2]). Let $\mathcal{E}:=\mathcal{O}_{X} \oplus \mathcal{O}_{X}(-D)$. Then we have an equivalence of triangulated categories

$$
\Phi:=\mathbf{R} f_{*} \mathbf{R} \mathcal{H o m}(\mathcal{E}, *): D^{b}(X) \stackrel{\cong}{\rightrightarrows} D^{b}(\operatorname{Coh}(\mathcal{A})),
$$

where $\mathcal{A}:=f_{*} \mathcal{E} n d(\mathcal{E})$. Furthermore, the functor $\Phi$ restricts to an equivalence $\operatorname{Per}(X / Y) \cong \operatorname{Coh}(\mathcal{A})$ of Abelian categories.

From the above theorem, we can easily get the following:

Lemma 2.3. Let $E \in \operatorname{Per}(X / Y)$. Then we have $\mathbf{R}^{1} f_{*}(E(D))=0$.

Proof. For $E \in \operatorname{Per}(X / Y)$, we have $\Phi(E)=\mathbf{R} f_{*} E \oplus \mathbf{R} f_{*}(E(D)) \in \operatorname{Coh}(\mathcal{A})$ by Theorem 2.2. This in particular implies $\mathbf{R}^{1} f_{*}(E(D))=0$.

We give a criterion when a coherent sheaf $E \in \operatorname{Coh}(X)$ is in $\operatorname{Per}(X / Y)$. Before stating the criterion, we recall the following lemma:

Lemma 2.4 ([13, Lemma 1.2]). Let $E$ be a coherent sheaf, $\phi: f^{*} f_{*} E \rightarrow E$ be the adjoint morphism. Then the following statements hold.

(1) We have $f_{*}($ Image $\phi) \cong f_{*} E$, and $\mathbf{R}^{1} f_{*}($ Image $\phi)=0$.

(2) We have $f_{*}($ Coker $\phi)=0$, and $\mathbf{R}^{1} f_{*}($ Coker $\phi) \cong \mathbf{R}^{1} f_{*} E$.

(3) $E \in \operatorname{Per}(X / Y)$ if and only if Coker $\phi=0$.

(4) We have $\operatorname{Ker}(\phi) \in \mathcal{C}^{0}$.

The following criterion will be frequently used in this paper:

Lemma 2.5 (cf. [13, Proposition 1.9]). Let $E$ be a coherent sheaf. Then $E$ is an object of the category $\operatorname{Per}(X / Y)$ if and only if for every point $y$ of $C$, we have $\operatorname{Hom}\left(E, \mathcal{O}_{L_{y}}(-1)\right)=0$, where $L_{y}:=f^{-1}(y) \cong \mathbb{P}^{1}$ and $\mathcal{O}_{L_{y}}(-1):=\mathcal{O}_{\mathbb{P}^{1}}(-1)$.

Proof. When $\operatorname{dim} Y=2$, the same statement is stated and proven by Nakajima and Yoshioka in [13, Proposition 1.9]. However, their proof does not work in the higher dimension. Hence we give the another proof which works in any dimension.

Assume that $E \in \operatorname{Per}(X / Y)$. Then by the definition of $\operatorname{Per}(X / Y)$, we have $\operatorname{Hom}\left(E, \mathcal{C}^{0}\right)=0$. In particular, we have $\operatorname{Hom}\left(E, \mathcal{O}_{L_{y}}(-1)\right)=0$.

For the converse, we have to show the following two things: 
(a) $\operatorname{Hom}\left(E, \mathcal{C}^{0}\right)=0$,

(b) $\mathbf{R}^{1} f_{*} E=0$.

First we prove (a). We need to show that $\operatorname{Hom}\left(E, \pi^{*} M \otimes \mathcal{O}(D)\right)$ vanishes for all $M \in \operatorname{Coh}(C)$. Take an element $\psi \in \operatorname{Hom}\left(E, \pi^{*} M \otimes \mathcal{O}(D)\right)$. For each point $y \in C$, consider the restriction

$$
\left.\psi\right|_{L_{y}}:\left.E\right|_{L_{y}} \rightarrow \pi^{*}\left(\left.M\right|_{\{y\}}\right) \otimes \mathcal{O}_{L_{y}}(D) \cong \mathcal{O}_{L_{y}}(-1)^{\oplus k}
$$

$\left(k \in \mathbb{Z}_{\geq 0}\right)$. By our assumption, $\left.\psi\right|_{L_{y}}$ is a zero map for all $y \in C$. Hence $\psi$ itself must be zero. This proves (a).

Next we prove (b). By the formal function theorem, it is enough to show that for every $y \in C$ and $n \in \mathbb{N}, H^{1}\left(L_{y, n}, E_{y, n}\right)=0$. Here, $L_{y, n}:=X \times_{Y} \operatorname{Spec} \mathcal{O}_{Y, y} / m_{y}^{n}$ and $E_{y, n}:=\left.E\right|_{L_{y, n}}$.

We argue by induction on $n$. First let $n=1$. To obtain a contradiction, suppose that there exists $y \in C$ such that $H^{1}\left(L_{y}, E_{y, 1}\right) \neq 0$. Let us consider the exact sequence

$$
0 \rightarrow\left(E_{y, 1}\right)_{t o r} \rightarrow E_{y, 1} \rightarrow\left(E_{y, 1}\right)_{f r} \rightarrow 0,
$$

where $\left(E_{y, 1}\right)_{t o r}$ (resp. $\left.\left(E_{y, 1}\right)_{f r}\right)$ is the torsion part (resp. torsion free part) of $E_{y, 1}$. Since $L_{y} \cong \mathbb{P}^{1}$, there exist integers $a_{i} \in \mathbb{Z}(i=1, \cdots, l)$ such that $\left(E_{y, 1}\right)_{f r} \cong$ $\bigoplus_{i=1}^{n} \mathcal{O}_{L_{y}}\left(a_{i}\right)$. Since $\bigoplus_{i=1}^{n} H^{1}\left(L_{y}, \mathcal{O}_{L_{y}}\left(a_{i}\right)\right) \cong H^{1}\left(L_{y}, E_{y, 1}\right) \neq 0$, there exists $i_{0}$ such that $a_{i_{0}} \leq-2$. On the other hand, by the surjection $E \rightarrow E_{y, 1} \rightarrow \mathcal{O}_{L_{y}}\left(a_{i_{0}}\right)$, we have

$$
\operatorname{Hom}\left(\mathcal{O}_{L_{y}}\left(a_{i_{0}}\right), \mathcal{O}_{L_{y}}(-1)\right) \subset \operatorname{Hom}\left(E, \mathcal{O}_{L_{y}}(-1)\right)=0,
$$

which is a contradiction. Hence when $n=1$, we have $H^{1}\left(L_{y}, E_{y, 1}\right)=0$ for every $y \in C$.

Next assume that for a fixed integer $n \in \mathbb{N}, H^{1}\left(L_{y, n}, E_{y, n}\right)=0$ holds $(y \in C)$. Consider the exact sequence

$$
0 \rightarrow m_{y}^{n} / m_{y}^{n+1} \rightarrow \mathcal{O}_{Y, y} / m_{y}^{n+1} \rightarrow \mathcal{O}_{Y, y} / m_{y}^{n} \rightarrow 0 .
$$

Note that $m_{y}^{n} / m_{y}^{n+1} \cong\left(\mathcal{O}_{Y, y} / m_{y}\right)^{\oplus k}$ for some $k \in \mathbb{N}$. Applying the functor $f^{*}(-) \otimes$ $E$ to the above exact sequence, we have the exact sequence

$$
E_{y, 1}^{\oplus k} \rightarrow E_{y, n+1} \rightarrow E_{y, n} \rightarrow 0 .
$$

Split this exact sequence into two short exact sequences:

$$
\begin{aligned}
& 0 \rightarrow M \rightarrow E_{y, 1}^{\oplus k} \rightarrow K \rightarrow 0, \\
& 0 \rightarrow K \rightarrow E_{n+1} \rightarrow E_{n} \rightarrow 0 .
\end{aligned}
$$

From the first exact sequence, we have the exact sequence

$$
0=H^{1}\left(X, E_{y, 1}\right)^{\oplus k} \rightarrow H^{1}(X, K) \rightarrow H^{2}(X, M)=0 .
$$

Note that $H^{1}\left(X, E_{y, 1}\right)=0$ follows from the argument of $n=1$ case, while $H^{2}(X, M)=0$ holds since $\operatorname{dim} \operatorname{Supp}(M) \leq 1$. Hence we also have $H^{1}(X, K)=0$. Then by the second exact sequence and the induction hypothesis, we conclude that $H^{1}\left(E_{y, n+1}\right)=0$ as required.

Corollary 2.6. Let $E \in \operatorname{Per}(X / Y) \cap \operatorname{Coh}(X)$ be a perverse coherent sheaf. Then $E(-D)$ is also a perverse coherent sheaf.

Proof. Take a point $y \in C$. Noting the isomorphism $\mathcal{O}_{L_{y}}(D) \cong \mathcal{O}_{L_{y}}(-1)$, we have

$$
\operatorname{Hom}\left(E(-D), \mathcal{O}_{L_{y}}(-1)\right)=\operatorname{Hom}\left(E, \mathcal{O}_{L_{y}}(-2)\right) \subset \operatorname{Hom}\left(E, \mathcal{O}_{L_{y}}(-1)\right)=0 .
$$

Hence by Lemma 2.5, we have $E(-D) \in \operatorname{Per}(X / Y)$. 
2.2. Moduli space of $m$-stable sheaves. In this subsection, we recall the notion of $m$-stability and the moduli space of $m$-stable sheaves introduced by Nakajima and Yoshioka in their paper 13 . Let $H$ be an ample divisor on $Y, v=\left(v_{0}, v_{1}, \cdots\right) \in$ $H^{2 *}(X ; \mathbb{Q})$ such that $v_{0}>0$ and $\operatorname{gcd}\left(v_{0}, v_{1} \cdot f^{*} H^{n-1}\right)=1$.

Definition 2.7. Let $E \in \operatorname{Coh}(X)$ be a coherent sheaf.

(1) We say that $E$ is 0 -stable if $E \in \operatorname{Per}(X / Y)$ and $f_{*} E \in \operatorname{Coh}(Y)$ is $\mu_{H}$-stable.

(2) Let $m \in \mathbb{Z}_{>0}$ be a positive integer. We say that $E$ is $m$-stable if $E(-m D)$ is 0 -stable.

Theorem 2.8 ([13, Theorem 2.9]). Let $m \in \mathbb{Z}_{\geq 0}$. Then there exists the projective coarse moduli scheme $M^{m}(v)$ of $m$-stable sheaves with Chern character $v$.

Remark 2.9. In [13, Section 2], the notion of $m$-stability and the existence of the coarse moduli space are discussed without assuming $\operatorname{gcd}\left(v_{0}, v_{1} \cdot f^{*} H^{n-1}\right)=1$. However, in the following, we use this assumption almost everywhere. In particular, the following fact will be used frequently: for $E \in \operatorname{Per}(X / Y) \cap \operatorname{Coh}(X)$ with $\operatorname{ch}(E)=v$, $f_{*} E$ is $\mu_{H}$-semistable if and only if it is $\mu_{H}$-stable.

\section{WALL-CROSSING}

In this section, we always fix an ample divisor $H$ on $Y$ and the Chern character $v=\left(v_{0}, v_{1}, \cdots\right) \in H^{2 *}(X ; \mathbb{Q})$ of a sheaf such that $v_{0}>0$ and $\operatorname{gcd}\left(v_{0}, v_{1}, f^{*} H^{n-1}\right)=$ 1. We will describe the difference between $m$-stability and $(m+1)$-stability. To do that, we may assume $m=0$ since we have an isomorphism

$$
(-) \otimes \mathcal{O}(-m D): M^{m}(v) \rightarrow M^{0}\left(v \cdot e^{-m D}\right) .
$$

3.1. Torsion pairs. To construct the diagram (1.1), we use torsion pairs (cf. [7).

Definition 3.1. Let $\mathcal{A}$ be an Abelian category, $\mathcal{T}, \mathcal{F} \subset \mathcal{A}$ full subcategories of $\mathcal{A}$. Then the pair $(\mathcal{T}, \mathcal{F})$ is a torsion pair on $\mathcal{A}$ if the following two conditions hold:

(1) $\operatorname{Hom}(\mathcal{T}, \mathcal{F})=0$.

(2) For every object $E \in \mathcal{A}$, there exists objects $T \in \mathcal{T}, F \in \mathcal{F}$, and an short exact sequence

$$
0 \rightarrow T \rightarrow E \rightarrow F \rightarrow 0
$$

Note that by the property (1), the exact sequence (2) is unique up to isomorphism. First we recall the following easy property of torsion pairs.

Lemma 3.2. Let $(\mathcal{T}, \mathcal{F})$ be a torsion pair on an Abelian category $\mathcal{A}$. Then $\mathcal{T}$ is closed under taking quotients.

Proof. Take an element $E \in \mathcal{T}$ and a surjective map $E \rightarrow Q$ in $\mathcal{A}$. By the definition of a torsion pair, there exists an exact sequence

$$
0 \rightarrow T \rightarrow Q \rightarrow F \rightarrow 0
$$

with $T \in \mathcal{T}, F \in \mathcal{F}$. Then we get a surjective map $E \rightarrow Q \rightarrow F$. On the other hand, we have $\operatorname{Hom}(\mathcal{T}, \mathcal{F})=0$ by definition. Hence we must have $F=0$, i.e. $Q=T \in \mathcal{T}$.

In this paper, we use the following two torsion pairs on $\operatorname{Coh}(X)$.

Definition 3.3. Define the full subcategories of $\operatorname{Coh}(X)$ as

$$
\begin{aligned}
\mathcal{T} & :=\left\{T \in \operatorname{Coh}(X): \mathbf{R}^{1} f_{*} T=0, \operatorname{Hom}\left(T, \mathcal{C}^{0}\right)=0\right\}, \\
\mathcal{F} & :=\left\{F \in \operatorname{Coh}(X): f_{*} F=0\right\} .
\end{aligned}
$$


Definition 3.4. We define the full subcategories $\mathcal{T}_{D}, \mathcal{F}_{D} \subset \operatorname{Coh}(X)$ as follows:

$$
\begin{aligned}
& \mathcal{T}_{D}:=\{T \in \operatorname{Coh}(X): T(-D) \in \operatorname{Per}(X / Y), \operatorname{Supp}(T) \subset D\}, \\
& \mathcal{F}_{D}:=\left\{F \in \operatorname{Coh}(X): \operatorname{Hom}\left(\mathcal{T}_{D}, F\right)=0\right\} .
\end{aligned}
$$

Lemma 3.5. The pairs $(\mathcal{T}, \mathcal{F})$ and $\left(\mathcal{T}_{D}, \mathcal{F}_{D}\right)$ are torsion pairs on $\operatorname{Coh}(X)$.

Proof. The assertion for the pair $(\mathcal{T}, \mathcal{F})$ has been proved for example in [13, Lemma 1.3]. For the pair $\left(\mathcal{T}_{D}, \mathcal{F}_{D}\right)$, it is enough to show that the subcategory $\mathcal{T}_{D} \subset$ $\operatorname{Coh}(X)$ is closed under taking quotients and extensions by [17, Lemma 2.15], since $\operatorname{Coh}(X)$ is noetherian. The conditions $\operatorname{Supp}(T) \subset D$ and $\operatorname{Hom}\left(T(-D), \mathcal{C}^{0}\right)=0$ are clearly closed under taking quotients and extensions. Furthermore, since the morphism $f: X \rightarrow Y$ has relative dimension one, we can also see that the condition $\mathbf{R}^{1} f_{*} T(-D)=0$ is closed under taking quotients and extensions.

Remark 3.6. Recall that we have $\operatorname{Per}(X / Y)=\langle\mathcal{F}[1], \mathcal{T}\rangle$. Similarly, we define

$$
\widetilde{\operatorname{Per}}(X / Y):=\left\langle\mathcal{F}_{D}[1], \mathcal{T}_{D}\right\rangle \text {. }
$$

By the general theory of torsion pairs and tilting, the category $\widetilde{\operatorname{Per}}(X / Y)$ is the heart of a bounded t-structure on $D^{b}(X)$ (cf. [7]).

Lemma 3.7. We have $\mathcal{C}^{0} \subset \mathcal{T}_{D}$. Furthermore, for a coherent sheaf $E \in \operatorname{Coh}(X)$ which is torsion free outside $D$, the following statements hold.

(1) The sheaf $f_{*}(E(-D))$ is torsion free if and only if $\operatorname{Hom}\left(\mathcal{T}_{D}, E\right)=0$, i.e. $E \in \mathcal{F}_{D}$.

(2) If the sheaf $f_{*}(E(-D))$ is torsion free, then $f_{*}(E)$ is also torsion free.

Proof. For the first assertion, recall from Lemma 2.1 that we have $\mathcal{C}^{0}=f^{*} \operatorname{Coh}(C) \otimes$ $\mathcal{O}(D)$. From this, we can see that $\operatorname{Supp}(T) \subset D$ for any element $T \in \mathcal{C}^{0}$. Moreover, we have $\mathcal{C}^{0} \otimes \mathcal{O}(-D) \cong f^{*} \operatorname{Coh}(C) \subset \mathcal{T}$. This proves the inclusion $\mathcal{C}^{0} \subset \mathcal{T}_{D}$.

(1) First assume that $\operatorname{Hom}\left(\mathcal{T}_{D}, E\right)=0$. Since we assume $E$ is torsion free outside $D$, it is enough to show that $\operatorname{Hom}\left(\operatorname{Coh}(C), f_{*}(E(-D))\right)=0$. We can compute as

$$
\begin{aligned}
\operatorname{Hom}\left(\operatorname{Coh}(C), f_{*}(E(-D))\right) & \cong \operatorname{Hom}\left(f^{*} \operatorname{Coh}(C), E(-D)\right) \\
& =\operatorname{Hom}\left(f^{*} \operatorname{Coh}(C) \otimes \mathcal{O}(D), E\right) \\
& =0 .
\end{aligned}
$$

Note that the last equality holds by the inclusion $\mathcal{C}^{0} \subset \mathcal{T}_{D}$.

For the converse, assume that $f_{*}(E(-D))$ is torsion free. Let $T \in \mathcal{T}_{D}$. Then

$$
\begin{aligned}
\operatorname{Hom}(T, E) & =\operatorname{Hom}(T(-D), E(-D)) \\
& \subset \operatorname{Hom}\left(f^{*} f_{*}(T(-D)), E(-D)\right) \\
& \cong \operatorname{Hom}\left(f_{*}(T(-D)), f_{*}(E(-D))\right) \\
& =0 .
\end{aligned}
$$

Note that the adjoint map $f^{*} f_{*}(T(-D)) \rightarrow T(-D)$ is surjective since $T(-D) \in$ $\operatorname{Per}(X / Y)$ by definition. Hence we have the inclusion $\operatorname{Hom}(T(-D), E(-D)) \subset$ $\operatorname{Hom}\left(f^{*} f_{*}(T(-D)), E(-D)\right)$. Note also that the last equality holds since $f_{*}(T(-D))$ is torsion.

(2) By (1), it is enough to show that $\operatorname{Hom}\left(\mathcal{T}_{D}, E(D)\right)=0$. Let $T \in \mathcal{T}_{D}$. Then we have $T(-D) \in \mathcal{T}_{D}$ by Corollary 2.6. Hence

$$
\operatorname{Hom}(T, E(D))=\operatorname{Hom}(T(-D), E)=0 .
$$

Here, the last equality holds again by (1). 


\subsection{From 0-stability to 1 -stability.}

Proposition 3.8. Let $E^{-} \in \operatorname{Coh}(X) \cap \operatorname{Per}(X / Y)$ be a perverse coherent sheaf with Chern character $\operatorname{ch}\left(E^{-}\right)=v$. Let

$$
0 \rightarrow T \rightarrow E^{-} \rightarrow F \rightarrow 0
$$

be the unique exact sequence in $\operatorname{Coh}(X)$ with $T \in \mathcal{T}_{D}, F \in \mathcal{F}_{D}$. Then the following hold:

(1) If $f_{*} E^{-}$is torsion free, then $T \in \mathcal{C}^{0}$.

(2) If $E^{-}$is 0 -stable, then $F$ is 0 -stable and 1-stable.

Proof. First we prove (1). Since $T(-D) \in \operatorname{Per}(X / Y)$, we have $\mathbf{R}^{1} f_{*} T=0$ by Lemma 2.3. On the other hand, we have the injection $0 \rightarrow f_{*} T \rightarrow f_{*} E^{-}$in $\operatorname{Coh}(Y)$. Since $T$ is supported on $D$ and $f_{*} E^{-}$is torsion free, we have $f_{*} T=0$. This proves (1).

Next assume that $E^{-}$is 0 -stable. Since we have a surjection $E^{-} \rightarrow F \rightarrow 0$ in $\operatorname{Coh}(X)$, we have $F \in \operatorname{Per}(X / Y) \cap \operatorname{Coh}(X)=\mathcal{T}$ by Lemma 3.2, Furthermore, the isomorphism $f_{*} E^{-} \cong f_{*} F$ implies $F$ is 0 -stable. It remains to show that $F$ is 1-stable. By Corollary 2.6, we have $F(-D) \in \operatorname{Per}(X / Y)$.

Claim 3.9. $f_{*}(F(-D))$ is torsion free.

Proof. Let $M \in \operatorname{Coh}(C)$. We must show that $\operatorname{Hom}\left(M, f_{*}(F(-D))\right)=0$. By Lemma 2.1 and Lemma 3.7, we have $f^{*} M \otimes \mathcal{O}(D) \in \mathcal{C}^{0} \subset \mathcal{T}_{D}$. Hence by adjunction, we get

$$
\operatorname{Hom}\left(M, f_{*}(F(-D))\right) \cong \operatorname{Hom}\left(f^{*} M \otimes \mathcal{O}(D), F\right)=0 .
$$

Now since $f_{*}(F(-D)) \rightarrow f_{*} F$ is an isomorpism outside $C$ and they are torsion free, it follows that $0 \rightarrow f_{*}(F(-D)) \rightarrow f_{*} F$ is injective in $\operatorname{Coh}(Y)$. Furthermore, by the facts that $f_{*} F$ is $\mu$-stable and $\mu\left(f_{*}(F(-D))\right)=\mu\left(f_{*} F\right)$, we conclude that $f_{*}(F(-D))$ is $\mu$-stable. This means that $F$ is 1 -stable.

Proposition 3.10. Let $F \in M^{0}(v) \cap M^{1}(v)$ be a 0-stable and 1-stable sheaf with Chern character $\operatorname{ch}(F)=v$. Let $T \in \mathcal{C}^{0}$ with a surjective map $\psi: F \rightarrow T[1] \rightarrow 0$ in $\operatorname{Per}(X / Y)$. Let $E^{-}:=\operatorname{Ker} \psi \in \operatorname{Per}(X / Y)$. Then $E^{-}$is 0 -stable but not 1-stable.

Proof. By shifting the exact triangle $E^{-} \rightarrow F \rightarrow T[1]$, we get an exact triangle $T \rightarrow E^{-} \rightarrow F$. Since $T, F \in \operatorname{Coh}(X)$, we also have $E^{-} \in \operatorname{Coh}(X)$. Furthermore, since $E^{-} \in \operatorname{Per}(X / Y)$ and $f_{*} E^{-} \cong f_{*} F, E^{-}$is 0 -stable. On the other hand, since $T \in \mathcal{C}^{0}$ and $\operatorname{Hom}\left(T, E^{-}\right) \neq 0, E^{-}$is not 1-stable by Lemma 3.7

\subsection{From 1-stability to 0-stability.}

Proposition 3.11. Let $E^{+} \in M^{1}(v)$ be a 1-stable sheaf with Chern character $\operatorname{ch}\left(E^{+}\right)=v$. Let

$$
0 \rightarrow F \rightarrow E^{+} \rightarrow T \rightarrow 0
$$

be the unique exact sequence in $\operatorname{Coh}(X)$ with $F \in \mathcal{T}, T \in \mathcal{F}$. Then we have

(1) $T \in \mathcal{C}^{0}$.

(2) $F$ is 0-stable and 1-stable.

Proof. (1) By definition, we have $f_{*} T=0$. On the other hand, since $E^{+}(-D) \in$ $\operatorname{Per}(X / Y) \cap \operatorname{Coh}(X)=\mathcal{T}$, it follows that $T(-D) \in \operatorname{Per}(X / Y)$ by Lemma 3.2. Hence by Lemma 2.3 , we get $\mathbf{R}^{1} f_{*} T=0$.

(2) By definition, $F \in \operatorname{Per}(X / Y)$. By Corollary 2.6, we also have $F(-D) \in$ $\operatorname{Per}(X / Y)$. First we will show that $F$ is 1 -stable. We have an exact sequence

$$
0 \rightarrow f_{*}(F(-D)) \rightarrow f_{*}\left(E^{+}(-D)\right) \rightarrow f_{*}(T(-D))
$$


in $\operatorname{Coh}(Y)$. Since $\operatorname{Supp}\left(f_{*}(T(-D))\right) \subset C$ and $f_{*}\left(E^{+}(-D)\right)$ is $\mu$-stable, it follows that $f_{*}(F(-D))$ is also $\mu$-stable. We conclude that $F$ is 1 -stable.

It remains to show that $F$ is 0 -stable. As in the argument of the previous proposition, it is enough to show that $f_{*} F$ is torsion free. But that follows from Lemma 3.7 (3).

Proposition 3.12. Let $F \in M^{0}(v) \cap M^{1}(v)$ be a 0 -stable and 1-stable sheaf with Chern character $\operatorname{ch}(F)=v$. Let $T \in \mathcal{C}^{0}$ with an injective map $0 \rightarrow T \rightarrow F[1]$ in $\widetilde{\operatorname{Per}}(X / Y)$. Let $E^{+}[1]$ be its cokernel. Then $E^{+}$is 1-stable but not 0-stable.

Proof. First note that $F \in \mathcal{F}_{D}$ and hence $F[1] \in \widetilde{\operatorname{Per}}(X / Y)$. Furthermore, $\mathcal{F}_{D}[1]$ is a torsion part of the torsion pair $\left(\mathcal{F}_{D}[1], \mathcal{T}_{D}\right)$ on $\widetilde{\operatorname{Per}}(X / Y)$. Hence $\mathcal{F}_{D}[1]$ is closed under taking quotients in the abelian category $\widetilde{\operatorname{Per}}(X / Y)$. In particular, $E^{+} \in \mathcal{F}_{D}$ and hence $f_{*}\left(E^{+}(-D)\right)$ is torsion free. Applying $f_{*}$ to the exact sequence

$$
0 \rightarrow F \rightarrow E^{+} \rightarrow T \rightarrow 0
$$

in $\operatorname{Coh}(X)$, we have an injection $0 \rightarrow f_{*}(F(-D)) \rightarrow f_{*}\left(E^{+}(-D)\right)$ which is isomorphism outside $C$. Hence the $\mu$-stability of $f_{*}(F(-D))$ implies that $f_{*}\left(E^{+}(-D)\right)$ is $\mu$-stable. On the other hand, since $T \in \mathcal{C}^{0}$ and $\operatorname{Hom}\left(E^{+}, T\right) \neq 0$, we have $E^{+} \notin \operatorname{Per}(X / Y)$. In particular, $E^{+}$is not 0-stable.

3.4. Set-theoretical wall-crossing. Define $\mathcal{S}:=\left\{\operatorname{ch}(T): T \in \mathcal{C}^{0}\right\}$. First we define the following two notions. Note that for a 0 -stable and 1-stable sheaf $F$, we have $F \in \operatorname{Per}(X / Y)$ by definition. Furthermore, by Lemma 3.7 we have $F \in \mathcal{F}_{D}$ and thus $F[1] \in \widetilde{\operatorname{Per}}(X / Y)$ (see Remark 3.6 for the definition of $\widetilde{\operatorname{Per}}(X / Y)$ ).

Definition 3.13. Let $F$ be a 0 -stable and 1 -stable sheaf and $\beta \in \mathcal{S}$. We define

$\operatorname{P}-\operatorname{Sub}(F, \beta):=\left\{0 \rightarrow E \rightarrow F \rightarrow T[1] \rightarrow 0\right.$ exact in $\left.\operatorname{Per}(X / Y): T \in \mathcal{C}^{0}, \operatorname{ch}(T)=\beta\right\}$,

$\widetilde{\mathrm{P}-\operatorname{Quot}}(F, \beta):=\left\{0 \rightarrow T \rightarrow F[1] \rightarrow E[1] \rightarrow 0\right.$ exact in $\left.\widetilde{\operatorname{Per}}(X / Y): T \in \mathcal{C}^{0}, \operatorname{ch}(T)=\beta\right\}$.

Summarizing the results in the previous subsections, we get:

Proposition 3.14. We have a diagram of sets

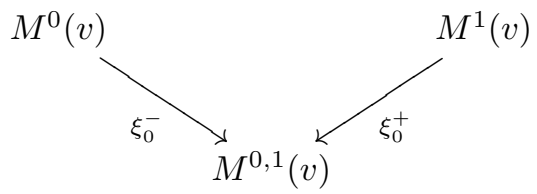

such that

- $M^{0,1}(v):=\coprod_{\beta \in \mathcal{S}}\left(M^{0}(v-\beta) \cap M^{1}(v-\beta)\right)$,

- the fibre of $F \in M^{0}(v-\beta) \cap M^{1}(v-\beta)$ over $\xi_{0}^{-}$is $\mathrm{P}-\operatorname{Sub}(F, \beta)$,

- the fibre of $F \in M^{0}(v-\beta) \cap M^{1}(v-\beta)$ over $\xi_{0}^{+}$is $\widetilde{\mathrm{P}-\operatorname{Quot}}(F, \beta)$.

Proof. We define the map $\xi_{0}^{-}$as follows: Take an element $E^{-} \in M^{0}(v)$, let $0 \rightarrow$ $T \rightarrow E^{-} \rightarrow F \rightarrow 0$ be the canonical exact sequence with $T \in \mathcal{T}_{D}, F \in \mathcal{F}_{D}$. Then $F$ is an element of $M^{0,1}(v)$ by Proposition [3.8. Hence we define $\xi_{0}^{-}\left(E^{-}\right):=F$. By the converse construction given in Proposition 3.10 , the fiber of $\xi_{0}^{-}$is given by $\operatorname{P-Sub}(F, \beta)$.

The map $\xi_{0}^{+}$is defined as follows: for an element $E^{+} \in M^{1}(v)$, we have an exact sequence $0 \rightarrow F \rightarrow E^{+} \rightarrow T \rightarrow 0$ with $F \in \mathcal{T}$ and $T \in \mathcal{F}$. Now we define as $\xi_{0}^{+}\left(E^{+}\right):=F$. Then the assersion follows from Proposition 3.11 and Proposition 3.12

In Section 5, we will construct the scheme-theoretic wall-crossing diagram. 


\section{Moduli space of Gieseker stable sheaves}

In this section, we will see the relationship between $m$-stability and the Gieseler stability on both $X$ and $Y$.

4.1. Moduli space of Gieseker stable sheaves on $Y$. Take $v=\left(v_{0}, v_{1}, \cdots\right) \in$ $H^{2 *}(X, \mathbb{Q})$ with $\operatorname{gcd}\left(f^{*} H^{n-1} \cdot v_{1}, v_{0}\right)=1$, and let $w:=f_{*}\left(v \cdot \operatorname{td}_{X}\right) \cdot \operatorname{td}_{Y}^{-1} \cdot$ Denote by $M^{H}(w)$ the moduli space of Gieseker $H$-stable sheaves on $Y$ with Chern character $v^{\prime}$. Then we have a morphism of schemes:

$$
\xi: M^{0}(v) \rightarrow M^{H}(w), E \mapsto \mathbf{R} f_{*} E=f_{*} E .
$$

Similarly, we have

$$
\xi^{+}: M^{1}(v) \rightarrow M^{H}(w), E \mapsto \mathbf{R} f_{*} E=f_{*} E .
$$

Note that $\xi^{+}$is well-defined. Indeed, take an element $E \in M^{1}(v)$. Then by Lemma 3.7. $f_{*} E$ is torsion free. Furthermore, since $f_{*} E(-D)$ is $\mu$-stable and is isomorphic to $f_{*} E$ in codimension $1, f_{*} E$ is also $\mu$-stable. Hence $\xi^{+}$is actually a morphism from $M^{1}(v)$ to $M^{H}(w)$.

Lemma 4.1. Let $F \in \operatorname{Coh}(Y)$ be a torsion free sheaf on $Y$. Then we have $\mathbf{L} f^{*} F=$ $f^{*} F$.

Proof. First we claim that $\mathbf{L} f^{*} F \in \operatorname{Per}(X / Y)$. We need to check the following three conditions (cf. [2, Lemma 3.2]):

(a) $\mathbf{R} f_{*}\left(\mathbf{L} f^{*} F\right) \in \operatorname{Coh}(Y)$,

(b) $\operatorname{Hom}\left(\mathbf{L} f^{*} F, \mathcal{C}^{>-1}\right)=0$,

(c) $\operatorname{Hom}\left(\mathcal{C}^{<-1}, \mathbf{L} f^{*} F\right)=0$.

(a), (b) are clear. We will show (c). Let $T \in \mathcal{C}^{0}, i \geq 1, f_{!}:=\mathbf{R} f_{*}\left(-\otimes \omega_{X}\right) \otimes \omega_{Y}^{-1}$. Then we have

$$
\operatorname{Hom}\left(T[i], \mathbf{L} f^{*} F\right) \cong \operatorname{Hom}(f ! T[i], F) .
$$

By the description of $f_{!}$, we know that $f_{!} T$ is a two term complex concentrated in degree 0 and 1 . Hence there exist $T^{\prime}, T^{\prime \prime} \in \operatorname{Coh}(Y)$ and an exact triangle

$$
T^{\prime}[i] \rightarrow f_{!} T[i] \rightarrow T^{\prime \prime}[i-1] .
$$

Applying $\operatorname{Hom}(-, F)$, we get an exact sequence

$$
\operatorname{Hom}\left(T^{\prime \prime}[i-1], F\right) \rightarrow \operatorname{Hom}(f ! T[i], F) \rightarrow \operatorname{Hom}\left(T^{\prime}[i], F\right) .
$$

Since $T^{\prime}, T^{\prime \prime}$ are torsion sheaves and $F$ is torsion free sheaf, we conclude that

$$
\operatorname{Hom}\left(T[i], \mathbf{L} f^{*} F\right) \cong \operatorname{Hom}\left(f_{!} T[i], F\right)=0 .
$$

Hence $\mathbf{L} f^{*} F \in \operatorname{Per}(X / Y)$.

Next we claim that $\mathbf{L}^{-1} f^{*} F \in \mathcal{C}^{0}$. Note that, if so, together with the equation (4.1), we must have $\mathbf{L}^{-1} f^{*} F=0$, i,e, $\mathbf{L} f^{*} F=f^{*} F$. By the spectral sequence

$$
\mathbf{R}^{p} \mathbf{L}^{q} f^{*} F \Rightarrow \mathcal{H}^{p+q}\left(\mathbf{R} f_{*} \mathbf{L} f^{*} F\right)=F,
$$

we have

- $f_{*}\left(\mathbf{L}^{-1} f_{*} F\right)=0$

- $0 \rightarrow \mathbf{R}^{1} f_{*}\left(\mathbf{L}^{-1} f^{*} F\right) \rightarrow F \rightarrow f_{*} f^{*} F \rightarrow 0$ is exact in $\operatorname{Coh}(Y)$.

Since $\mathbf{R}^{1} f_{*}\left(\mathbf{L}^{-1} f^{*} F\right)$ is a torsion sheaf and we assume $F$ is torsion free, we have $\mathbf{R}^{1} f_{*}\left(\mathbf{L}^{-1} f^{*} F\right)=0$. This proves that $\mathbf{L} f^{*} F=f^{*} F$.

Corollary 4.2. Assume that $v \in f^{*} H^{*}(Y ; \mathbb{Q}) \subset H^{*}(X ; \mathbb{Q})$. Then the morphism $\xi$ is an isomorphism. 
Proof. By Lemma 4.1, we can define a morphism $\eta$ as

$$
\eta: M^{H}(w) \rightarrow M^{0}(v), F \mapsto \mathbf{L} f^{*} F=f^{*} F .
$$

Then by the projection formula, we have $\xi \circ \eta=$ id. On the other hand, let $E \in M^{0}(v)$. Then we have an exact sequence

$$
0 \rightarrow K \rightarrow f^{*} f_{*} E \rightarrow E \rightarrow 0
$$

in $\operatorname{Coh}(X)$, since $E \in \operatorname{Per}(X / Y)$. By Lemma2.4(4), we have $K \in \mathcal{C}^{0}$. In particular, $\operatorname{ch}(K) \notin f^{*} H^{*}(Y ; \mathbb{Q})$. Since we assume $v \in f^{*} H^{*}(Y ; \mathbb{Q})$, we must have $K=0$, i.e, $\eta \circ \xi=\mathrm{id}$.

4.2. Moduli space of Gieseker stable sheaves on $X$. First we recall the finiteness result of walls for $\mu$-stability:

Proposition 4.3 (cf. [16, Lemma 1.1.7]). Let $H$ be an ample divisor on $Y$, take $\epsilon_{0} \in \mathbb{Q}_{>0}$ so that $f^{*} H-\epsilon_{0} D$ is ample on $X$. Let

$$
\Delta:=\left\{H_{\epsilon}:=f^{*} H-\epsilon D: 0 \leq \epsilon \leq \epsilon_{0}\right\} .
$$

Then there exist only finitely many walls on $\Delta$ for $\mu$-stability with respect to $v$.

Proof. The argument is essentially same as [16, Lemma 1.1.7]. However, since $f^{*} H$ is not ample, we need to be a little bit careful. The proof needs the following two facts:

(1) The Bogomolov-Gieseker (BG) inequality for $\mu_{H_{\epsilon}}$-stable sheaves.

(2) Let $x \in \mathrm{NS}(X)_{\mathbb{R}}$. If $x \cdot H_{\epsilon}^{n-1}=0$, then $-x^{2} \cdot H_{\epsilon}^{n-2} \geq 0$.

Firstly, (1) holds since the BG inequality holds for all nef divisor (cf. [10]). Next consider the statement (2). If $\epsilon \neq 0$, then $H_{\epsilon}$ is ample and the statement holds by the Hodge index theorem. Assume $\epsilon=0$, let $x_{\epsilon}:=x-\frac{x \cdot H_{\epsilon}^{n-1}}{D \cdot H_{\epsilon}^{n-1}} D$. Then

$$
x_{\epsilon} \cdot H_{\epsilon}^{n-1}=x \cdot H_{\epsilon}^{n-1}-\frac{x \cdot H_{\epsilon}^{n-1}}{D \cdot H_{\epsilon}^{n-1}} D \cdot H_{\epsilon}^{n-1}=0
$$

and $\lim _{\epsilon \rightarrow+0} x_{\epsilon}=x$. Since $x_{\epsilon}$ changes continuously with respect to $\epsilon$, we get the required result from the result of the ample case.

By Proposition 4.3 there exist $0<\epsilon(v)$ such that for every $0<\epsilon \leq \epsilon(v)$, $M^{f^{*} H-\epsilon D}(v)$ is constant.

Proposition 4.4. There exists an integer $m(v)>0$ such that for every $m \geq m(v)$, we have an open and closed embedding $M^{f^{*} H-\epsilon(v) D}(v) \subset M^{m}(v)$.

Proof. It is enough to show the following: there exists $m(v)>0$ such that for every $m \geq m(v)$ and $E \in M^{f^{*} H-\epsilon(v) D}(v)$, we have $E \in M^{m}(v)$. Indeed, if so, we have an immersion $\Phi: M^{f^{*} H-\epsilon(v) D}(v) \hookrightarrow M^{m}(v)$. By the openness of $\mu$-stability, $\Phi$ is an open immersion. On the other hand, since both $M^{f^{*} H-\epsilon(v) D}(v)$ and $M^{m}(v)$ are projective, $\Phi$ is also a closed immersion.

First of all, since $M^{f^{*} H-\epsilon(v) D}(v)$ is bounded, there exists a fixed sheaf $U \in$ $\operatorname{Coh}(X)$ such that for every $E \in M^{f^{*} H-\epsilon(v) D}(v)$, we have a surjective map $U \rightarrow E$ (cf. [9, Lemma 1.7.6]). On the other hand, since $-D$ is $f$-ample, there exists an integer $m(v)>0$ such that for every $m \geq m(v)$, the adjoint map $f^{*} f_{*}(U(-m D)) \rightarrow$ $U(-m D)$ is surjective. Then we have a commutative diagram

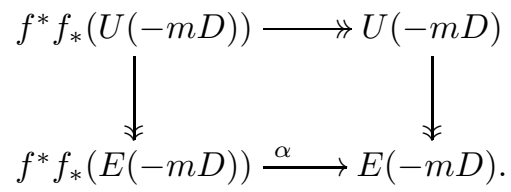


Hence the adjoint map $\alpha$ is also surjective, i.e. $E(-m D) \in \operatorname{Per}(X / Y)$.

It remains to show that for $m \geq m(v), f_{*}(E(-m D))$ is $\mu_{H}$-stable. Note that $f_{*}(E(-m D))$ is torsion free since so is $E$. Take a non-zero proper subsheaf $F \subset$ $f_{*}(E(-m D))$, and let $\phi: f^{*} F \rightarrow E(-m D)$ be the corresponding map. Taking its cone $T:=\operatorname{Cone}(\phi)$, we have

$$
\begin{aligned}
& 0 \rightarrow \mathcal{H}^{-1}(T) \rightarrow f^{*} F \rightarrow Q \rightarrow 0, \\
& 0 \rightarrow Q \rightarrow E(-m D) \rightarrow \mathcal{H}^{0}(T) \rightarrow 0 .
\end{aligned}
$$

Here, $\mathcal{H}^{i}(T)$ denotes the cohomology with respect to the heart $\operatorname{Coh}(X)$ for each integer $i \in \mathbb{Z}$. Note that since $\phi$ is injective outside $D, \operatorname{Supp}\left(\mathcal{H}^{-1}(T)\right) \subset D$. Hence we can write $\operatorname{ch}\left(\mathcal{H}^{-1}(T)\right)=(0, l D, \cdots)$ with $l \geq 0$. Now by the $\mu_{f * H-\epsilon(v) D^{\text {-stability }}}$ of $E(-m D)$, we have

$$
\mu_{f^{*} H-\epsilon D}(Q)<\mu_{f^{*} H-\epsilon D}(E(-m D))
$$

for all $0<\epsilon \leq \epsilon(v)$. Taking the limit $\epsilon \rightarrow+0$, we have

$$
\mu_{H}(F)=\mu_{f^{*} H}\left(f^{*} F\right)=\mu_{f^{*} H}(Q) \leq \mu_{f^{*} H}(E(-m D))=\mu_{H}\left(f_{*}(E(-m D))\right) .
$$

This shows that $E \in M^{m}(v)$.

Remark 4.5. As mentioned in the introduction, when $n=\operatorname{dim} Y=2$, the embedding given in Proposition 4.4 is actually an isomorphism (see Theorem 1.2). When $n \geq 3$, we do not know whether the inclusion is isomorphism or not in general. However, see the following example.

Example 4.6. Let $n=3, v=(1,0,0,-k) \in H^{2 *}(X ; \mathbb{Q})$ with $k \in \mathbb{Z}_{>0}$. We claim that for a sufficiently large integer $m \in \mathbb{Z}$, we have an isomorphism between $M^{m}(v)$ and $M^{f^{*} H-\epsilon D}(v)$. By Proposition 4.4, it is enough to show that every $m$-stable sheaf $E \in M^{m}(v)$ is torsion free. Let us consider the exact sequence

$$
0 \rightarrow E_{t o r} \rightarrow E \rightarrow E_{f r} \rightarrow 0,
$$

where $E_{t o r}$ (resp. $E_{f r}$ ) is the torsion (resp. torsion free) part of E.

First assume that $\operatorname{dim} \operatorname{Supp}\left(E_{\text {tor }}\right)=2$. Take a general member $A$ in the linear system $|H|$ and let $f_{A}: A^{\prime}:=f^{-1}(A) \rightarrow A$. We may assume that

(1) $\left.\left(f_{*} E(-m D)\right)\right|_{A} \cong f_{A *}\left(\left.E(-m D)\right|_{A^{\prime}}\right)$ is torsion free.

(2) $\left.E_{t o r}\right|_{A^{\prime}} \neq 0$.

This means $\left.E\right|_{A^{\prime}}$ is an $m$-stable sheaf on the smooth projective surface $A^{\prime}$ which has a non-trivial torsion part. Hence by Theorem $1.2(3), m$ is bounded from above.

We may now assume that $\operatorname{dim} \operatorname{Supp}\left(E_{\text {tor }}\right)=1$. Since $f_{*}(E(-m D))$ is torsion free, we can see that

$$
E_{\text {tor }} \in\left\langle\mathcal{O}_{L_{y}}\left(a_{y}\right): y \in C, \quad a_{y}+m<0\right\rangle .
$$

In particular, we have $\operatorname{ch}\left(E_{f r}\right)=\left(1,0,-\sum_{i=1}^{l} n_{i} L_{y_{i}},-k-\sum_{i=1}^{l} n_{i}\left(a_{i}+\frac{1}{2}\right)\right)$, where $y_{i} \in C, a_{i}+m<0$. Now consider the exact sequence

$$
0 \rightarrow E_{f r} \rightarrow\left(E_{f r}\right)^{D D} \cong \mathcal{O}_{X} \rightarrow \mathcal{O}_{Z} \rightarrow 0 .
$$

Here we use the speciality of our choice of $v$, which implies that $\left(E_{f r}\right)^{D D} \cong \mathcal{O}_{X}$ for any $E \in M^{m}(v)$. Since $\mathbf{R} f_{*} \mathcal{O}_{Z}=f_{*} \mathcal{O}_{Z}$ is zero dimensional, the Riemann-Roch theorem yeilds

$$
0 \leq \chi\left(\mathbf{R} f_{*} \mathcal{O}_{Z}\right)=k+\sum_{i=1}^{l} n_{i}\left(a_{i}+\frac{1}{2}\right) .
$$

Since $a_{i}+m<0$, we get the inequality $k \geq m\left(\sum_{i=1}^{l} n_{i}\right)$ which bounds $m$ from above. 


\section{Scheme StruCture on $M^{0,1}(v)$}

In this section, we define the scheme structure on $M^{0,1}(v)$ connecting $M^{0}(v)$ and $M^{1}(v)$. Recall that we have constructed a set-theoretic diagram in Proposition 3.14

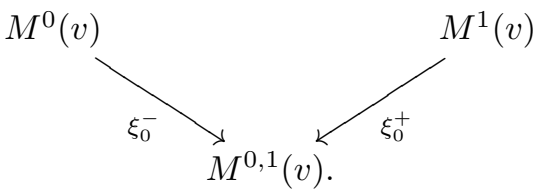

On the other hand, we have a scheme-theoretic diagram

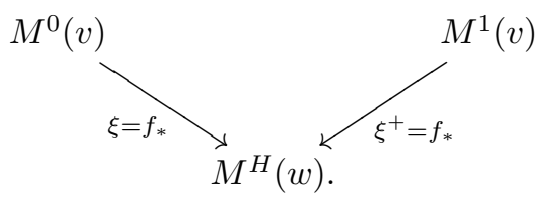

In the following, we will show that these two diagrams are essentially same.

Proposition 5.1.

(1) The morphism

$$
\left.\xi\right|_{M^{0}(v) \cap M^{1}(v)}: M^{0}(v) \cap M^{1}(v) \rightarrow M^{H}(w)
$$

is an immersion.

(2) We can identify $\xi_{0}^{-}\left(M^{0}(v)\right)$ with $\xi\left(M^{0}(v)\right)$.

(3) Under the identification (2), we have $\xi=\xi_{0}^{-}$.

Proof. (1) Since $M^{H}(w)$ is a projective scheme, it is enough to show that the morphism is injective and it induces injection between tangent spaces.

First we will show that the morphism (5.3) is injective. Let $E, E^{\prime} \in M^{0}(v) \cap$ $M^{1}(v)$ with $\phi: f_{*} E^{\prime} \cong f_{*} E$. Then we have the commutative diagram

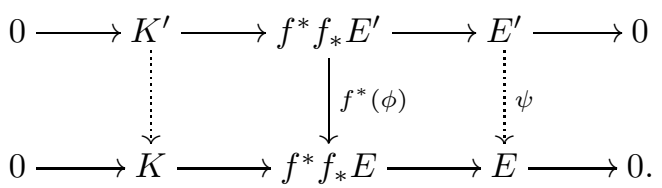

Note that since $K^{\prime} \in \mathcal{C}^{0}$ and $E$ is 1-stable, we have $\operatorname{Hom}\left(K^{\prime}, E\right)=0$. Hence the composition $K^{\prime} \rightarrow f^{*} f_{*} E^{\prime} \rightarrow f^{*} f_{*} E$ factors through $K^{\prime} \rightarrow K \rightarrow f^{*} f_{*} E$. As a result, we get an exact sequence

$$
0 \longrightarrow T \longrightarrow E^{\prime} \stackrel{\psi}{\longrightarrow} E \longrightarrow 0 \text {. }
$$

Applying $\mathbf{R} f_{*}$ to the diagram (5.4), we have $\mathbf{R} f_{*}(\psi)=\phi: f_{*} E^{\prime} \cong f_{*} E$ and hence $T \in \mathcal{C}^{0}$. Since $E^{\prime}$ is 1 -stable, $T$ must be 0 , i.e. $\psi: E^{\prime} \cong E$.

It remains to show that for every $E \in M^{0}(v) \cap M^{1}(v)$, there exists an inclusion between tangent spaces

$$
T_{[E]} M^{0}(v)=\operatorname{Ext}^{1}(E, E) \stackrel{f_{*}}{\longrightarrow} \operatorname{Ext}^{1}\left(f_{*} E, f_{*} E\right)=T_{\left[f_{*} E\right]} M^{H}(w) .
$$

Note that since $E \in M^{0}(v) \cap M^{1}(v)$, we have $\mathbf{R} f_{*} E=f_{*} E$ and $\mathbf{L} f^{*} \mathbf{R} f_{*} E \cong f^{*} f_{*} E$ (see Lemma 4.1). Applying $\operatorname{Hom}(-, E)$ to the second row of the diagram (5.4), we get

$$
0=\operatorname{Hom}(K, E) \rightarrow \operatorname{Ext}^{1}(E, E) \hookrightarrow \operatorname{Ext}^{1}\left(f^{*} f_{*} E, E\right) \cong \operatorname{Ext}^{1}\left(f_{*} E, f_{*} E\right)
$$


Note that $\operatorname{Hom}(K, E)=0$ follows from the 1-stability of $E$. By [ 8 , Lemma 1.21], the diagram

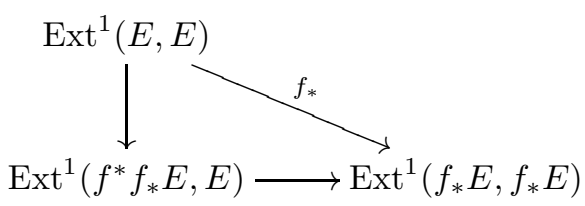

is commutative. Hence we conclude that the tangent map $f_{*}$ is injective.

(2) For $G=\xi(E) \in \xi\left(M^{0}(v)\right)$, we associate an element $\Phi(G)$ of $\xi_{0}^{-}\left(M^{0}(v)\right)$ as follows: we have a commutative diagram

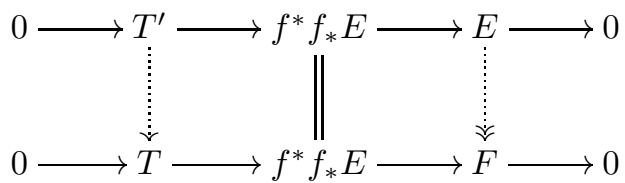

in $\operatorname{Coh}(X)$, where $T^{\prime}$ is the kernel of the adjoint map and the bottom sequence is the decomposition with respect to the torsion pair $\left(\mathcal{T}_{D}, \mathcal{F}_{D}\right)$. Since $f^{*} f_{*} E$ is 0 -stable, we have $T \in \mathcal{C}^{0}$ and $F$ is 1 -stable by Proposition 3.8. Note that since $\operatorname{Hom}\left(\mathcal{C}^{0}, F\right)=0$, we have a surjective map $E \rightarrow F \rightarrow 0$. Furthermore, by the construction, its kernel $\operatorname{Ker}(E \rightarrow F) \in \mathcal{C}^{0}$. In other words,

$$
\Phi(G):=F=\xi_{0}^{-}(E) \in \xi_{0}^{-}\left(M^{0}(v)\right) .
$$

Note that the definition of $\Phi$ is independent of the choice of $E \in M^{0}(v)$ with $f_{*} E=G$, i.e. the map $\Phi$ is well-defined. Indeed, $F$ only depends on $f^{*} f_{*} E=f^{*} G$.

Claim 5.2. The map $\Phi$ is injective.

Proof. Let $G, G^{\prime} \in \xi\left(M^{0}(v)\right)$ with $\Phi(G)=\Phi\left(G^{\prime}\right)$. Then we have $G=f_{*} \Phi(G)=$ $f_{*} \Phi\left(G^{\prime}\right)=G^{\prime}$.

Claim 5.3. The map $\Phi$ is surjective.

Proof. Let $F=\xi_{0}^{-}(E) \in \xi_{0}^{-}\left(M^{0}(v)\right)$. Then the equation (5.5) shows that $F=$ $\Phi(\xi(E))$.

(3) The assertion follows from the equation (5.5).

Proposition 5.4. $\quad(1)$ We can identify $\xi_{0}^{+}\left(M^{1}(v)\right)$ with $\xi^{+}\left(M^{1}(v)\right)$.

(2) Via the identification (1), we have $\xi^{+}=\xi_{0}^{+}$.

Proof. The proof is similar to that of Proposition 5.1. Hence we just give an outline of the proof. We define the map $\Psi: \xi^{+}\left(M^{1}(v)\right) \rightarrow \xi_{0}^{+}\left(M^{1}(v)\right)$ as follows: Let $F=\xi^{+}(E) \in \xi^{+}\left(M^{1}(v)\right)$. Then we have $\phi: f_{*} E \cong F$ by definition. Take an element $\alpha \in \operatorname{Hom}\left(E, f^{*} F(D)\right)$ corresponding to $\phi$ via the isomorphism $\operatorname{Hom}\left(f_{*} E, F\right) \cong$ $\operatorname{Hom}\left(E, f^{*} F(D)\right)$. Let $C:=\operatorname{Cone}(\alpha)$. Then since $f_{*}(\alpha)=\phi$, we have $C \in \mathcal{C}$. Furthermore, since $E$ is 1 -stable, we must have $\mathcal{H}^{-1}(C)=0$, i.e. $C$ is a sheaf. Hence we have the exact sequence

$$
0 \longrightarrow E \stackrel{\alpha}{\longrightarrow} f^{*} F(D) \longrightarrow C \longrightarrow 0
$$


in $\operatorname{Coh}(X)$. Consider the following commutative diagram

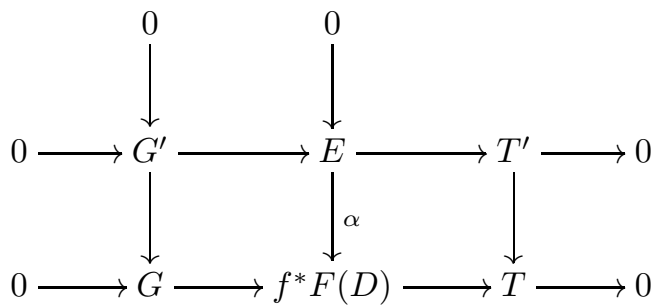

with $G^{\prime}, G \in \mathcal{T}$ and $T^{\prime}, T \in \mathcal{F}$. Using the fact that $C=\operatorname{Coker}(\alpha) \in \mathcal{C}^{0}$, we can show that $G^{\prime} \cong G$. Now we define as $\Psi(F):=G=G^{\prime}=\xi_{0}^{+}(E) \in \xi_{0}^{+}\left(M^{1}(v)\right)$. As in Proposition 5.1, we can prove that $\Psi$ is bijection. Furthermore, the second assertion follows from the definition of the map $\Psi$.

As a summary of Proposition 3.14, Proposition 5.1, and Proposition 5.4, we get:

Proposition 5.5. There exists a diagram of projective schemes

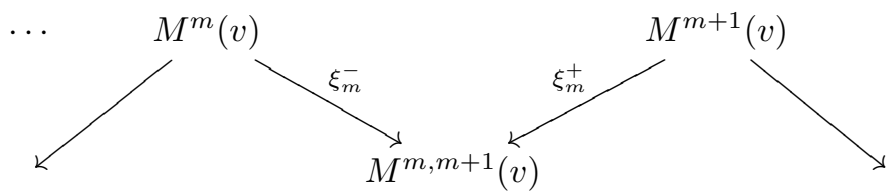

connecting the moduli spaces of $m$-stable sheaves, where the morphisms $\xi_{m}^{ \pm}$are defined as $\xi_{m}^{ \pm}:=f_{*}(-\otimes \mathcal{O}(-m D))$. Moreover, we can also describe the morphisms $\xi_{m}^{+}, \xi_{m}^{-}$in terms of the decompositions with respect to the torsion pairs $(\mathcal{T} \otimes \mathcal{O}(m D), \mathcal{F} \otimes \mathcal{O}(m D)),\left(\mathcal{T}_{D} \otimes \mathcal{O}(m D), \mathcal{F}_{D} \otimes \mathcal{O}(m D)\right)$, respectively

We end with the following proposition describing the fibers of $\xi$ and $\xi^{+}$. We can think them as the scheme structures on $\operatorname{P-Sub}(F, \beta)$ and $\widetilde{\mathrm{P}-\mathrm{Quot}}(F, \beta)$ defined in Definition 3.13

Proposition 5.6. Let $F \in M^{H}(w)$ be a $H$-stable sheaf with Chern character $\operatorname{ch}(F)=v^{\prime}$. Then the following statements hold.

(1) We have $\xi^{-1}(F)=\operatorname{Quot}\left(f^{*} F, v\right)$.

(2) We have $\left(\xi^{+}\right)^{-1}(F)=\operatorname{Sub}\left(f^{*} F(D), v\right)$.

Proof. (1) Let $E \in \xi^{-1}(F)$. Then we have an exact sequence

$$
0 \rightarrow T \rightarrow f^{*} F=f^{*} f_{*} E \rightarrow E \rightarrow 0
$$

in $\operatorname{Coh}(X)$, i.e. $E \in \operatorname{Quot}\left(f^{*} F, v\right)$.

For the converse, let $E \in \operatorname{Quot}\left(f^{*} F, v\right)$. Then we have

$$
0 \rightarrow K \rightarrow f^{*} F \rightarrow E \rightarrow 0 \text {. }
$$

Note that $E \in \operatorname{Per}(X / Y)$. Since $K$ is torsion and $F$ is torsion free, we have $f_{*} K=0$ and $F \hookrightarrow f_{*} E$ is injective. Moreover, since $\operatorname{ch}(F)=\operatorname{ch}\left(f_{*} E\right)$, we have $F \cong f_{*} E$. Hence $E \in \xi^{-1}(F)$. Noting that $\operatorname{Hom}\left(f^{*} F, E\right) \cong \operatorname{Hom}\left(F, f_{*} E\right)=\mathbb{C}$, we conclude that $\xi^{-1}(F)=\operatorname{Quot}\left(f^{*} F, v\right)$.

(2) Let $E \in\left(\xi^{+}\right)^{-1}(F)$. Then by the proof of Proposition 5.4, we have the exact sequence as in (5.6):

$$
0 \rightarrow E \rightarrow f^{*} F(D) \rightarrow C \rightarrow 0
$$

In other words, $E \in \operatorname{Sub}\left(f^{*} F(D), v\right)$.

For the converse, let $E \in \operatorname{Sub}\left(f^{*} F(D), v\right)$. Then we have

$$
0 \rightarrow E \rightarrow f^{*} F(D) \rightarrow C \rightarrow 0 \text {. }
$$


First we claim that $C \in \mathcal{C}^{0}$. Since $f^{*} F \in \operatorname{Per}(X / Y)$, we have $C(-D) \in$ $\operatorname{Per}(X / Y)$ by Lemma 3.2. Hence by Lemma 2.3, we have $\mathbf{R}^{1} f_{*} C=0$. Furthermore, we have $\operatorname{ch}(C) \in \mathcal{S}$. Hence $\operatorname{ch}\left(f_{*} C\right)=\operatorname{ch}\left(\mathbf{R} f_{*} C\right)=0$ and so we also have $f_{*} C=0$.

Next we show that $E(-D) \in \operatorname{Per}(X / Y)$. To show this, it is enough to show that for $y \in C$, we have $\operatorname{Hom}\left(E(-D), \mathcal{O}_{L_{y}}(-1)\right)=0$. Write $C(-D)=f^{*} M$. Applying $\operatorname{Hom}\left(-, \mathcal{O}_{L_{y}}(-1)\right)$ to the exact sequence

$$
0 \rightarrow E(-D) \rightarrow f^{*} F \rightarrow f^{*} M \rightarrow 0
$$

we have

$$
0=\operatorname{Hom}\left(f^{*} F, \mathcal{O}_{L_{y}}(-1)\right) \rightarrow \operatorname{Hom}\left(E(-D), \mathcal{O}_{L_{y}}(-1)\right) \rightarrow \operatorname{Hom}\left(f^{*} M, \mathcal{O}_{L_{y}}(-1)[1]\right) .
$$

Hence it is enough to show that $\operatorname{Hom}\left(f^{*} M, \mathcal{O}_{L_{y}}(-1)[1]\right)=0$. From the exact triangle

$$
\mathbf{L}^{-1} f^{*} M[1] \rightarrow \mathbf{L} f^{*} M \rightarrow f^{*} M
$$

we have

$$
\begin{aligned}
0=\operatorname{Hom}\left(\mathbf{L}^{-1} f^{*} M[1], \mathcal{O}_{L_{y}}(-1)\right) & \rightarrow \operatorname{Hom}\left(f^{*} M, \mathcal{O}_{L_{y}}(-1)[1]\right) \\
& \rightarrow \operatorname{Hom}\left(\mathbf{L} f^{*} M, \mathcal{O}_{L_{y}}(-1)[1]\right) \\
& \cong \operatorname{Hom}\left(M, \mathbf{R} f_{*} \mathcal{O}_{L_{y}}(-1)[1]\right) \\
& =0 .
\end{aligned}
$$

As a conclusion, we have $E(-D) \in \operatorname{Per}(X / Y)$. Furthermore, since $f^{*} F(D) \in$ $\mathcal{F}_{D}$, we also have $E \in \mathcal{F}_{D}$. Hence by Lemma 3.7. $f_{*} E(-D)$ is torsion free. Since $f_{*} E \cong F$ is $\mu$-stable, we conclude that $f_{*} E(-D)$ is also $\mu$-stable, i.e. $E \in$ $\left(\xi^{+}\right)^{-1}(F)$. As before, noting that $\operatorname{Hom}\left(E, f^{*} F(D)\right) \cong \operatorname{Hom}\left(f_{*} E, F\right)=\mathbb{C}$, we have $\left(\xi^{+}\right)^{-1}(F)=\operatorname{Sub}\left(f^{*} F(D), v\right)$.

\section{HilberT SCHEME OF TWO POINTS}

In this section, we study the birational geometry of Hilbert scheme of two points using the flip-like diagram (1.1) constructed in the previous sections. In the followings, we assume that $H^{1}\left(\mathcal{O}_{Y}\right)=0$ and let $w:=(1,0, \cdots, 0,-2) \in H^{*}(Y ; \mathbb{Q}), v:=$ $f^{*} w \in H^{*}(X ; \mathbb{Q})$. Then we have $M^{H}(w)=\operatorname{Hilb}^{2}(Y), M^{f^{*} H-\epsilon D}(v)=\operatorname{Hilb}^{2}(X)$. We will use that following notations:

- For a 0-dimensional closed subscheme $Z \subset X$ of length 2 , we denote its ideal sheaf as $I_{Z} \in \operatorname{Hilb}^{2}(X)$.

- $\operatorname{Hilb}^{2}(D / C) \subset \operatorname{Hilb}^{2}(X)$ denotes the relative Hilbert scheme which parametrizes $I_{Z} \in \operatorname{Hilb}^{2}(X)$ such that $Z$ is scheme-theoretically contained in a fiber of $\pi: D \rightarrow C$, i.e. there exists $y \in C$ such that $Z \subset L_{y}$.

- $\mathcal{I} \in \operatorname{Coh}\left(\operatorname{Hilb}^{2}(X) \times X\right)$ denotes the universal ideal sheaf on $\operatorname{Hilb}^{2}(X)$.

We start with the following lemma:

Lemma 6.1. Let $I_{Z} \in \operatorname{Hilb}^{2}(X)$ be an ideal sheaf of a length two closed subscheme $Z \subset X$. Then the following holds:

(1) If $Z \cap D=\emptyset$, then $I_{Z}$ is 0-stable.

(2) If $Z \cap D \neq \emptyset$ and $I_{Z} \notin \operatorname{Hilb}^{2}(D / C)$, then $I_{Z}$ is 1-stable but not 0 -stable.

(3) If $I_{Z} \in \operatorname{Hilb}^{2}(D / C)$, then $I_{Z}$ is 2-stable but not 1-stable.

Proof. By the proof of Proposition 4.4 it is enough to find the smallest $m \in \mathbb{Z}_{\geq 0}$ such that $I_{Z}(-m D) \in \operatorname{Per}(X / Y)$, i.e.

$$
\operatorname{Hom}\left(I_{Z}, \mathcal{O}_{L_{y}}(-m-1)\right)=\operatorname{Hom}\left(I_{Z}(-m D), \mathcal{O}_{L_{y}}(-1)\right)=0
$$

for all $y \in C$. 
Restricting the exact sequence

$$
0 \rightarrow I_{Z} \rightarrow \mathcal{O}_{X} \rightarrow \mathcal{O}_{Z} \rightarrow 0
$$

to $L_{y}$, we get

$$
\left.0 \rightarrow T \rightarrow I_{Z}\right|_{L_{y}} \rightarrow \mathcal{O}_{L_{y}} \rightarrow \mathcal{O}_{Z \cap L_{y}} \rightarrow 0,
$$

where $T \in \operatorname{Coh}\left(L_{y}\right)$ is some torsion sheaf. Hence we get

$$
\left.0 \rightarrow T \rightarrow I_{Z}\right|_{L_{y}} \rightarrow \mathcal{O}_{L_{y}}(-l) \rightarrow 0,
$$

where $l \in \mathbb{Z}$ is the length of $Z \cap L_{y}$. We conclude that

$$
\begin{aligned}
\operatorname{Hom}\left(I_{Z}, \mathcal{O}_{L_{y}}(-m-1)\right) & =\operatorname{Hom}\left(\left.I_{Z}\right|_{L_{y}}, \mathcal{O}_{L_{y}}(-m-1)\right) \\
& =\operatorname{Hom}\left(\mathcal{O}_{L_{y}}(-l), \mathcal{O}_{L_{y}}(-m-1)\right) .
\end{aligned}
$$

Hence we get the result.

Corollary 6.2. The exceptional locus of $\xi_{1}^{+}$is $\operatorname{Exc}\left(\xi_{1}^{+}\right)=\operatorname{Hilb}^{2}(D / C)$.

Proof. The assertion directly follows from Lemma 6.1

By Lemma 6.1, we have the diagram:

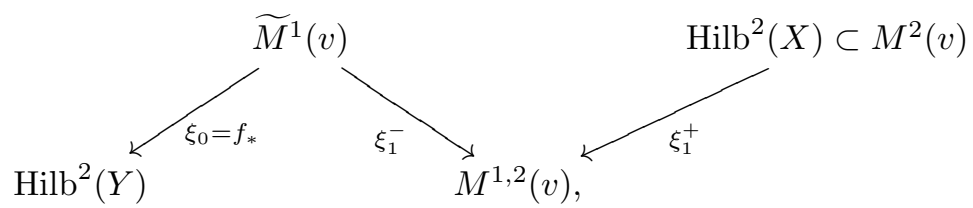

where $\widetilde{M}^{1}(v)$ denotes the normalization of the connected component of $M^{1}(v)$ containing $\operatorname{Hilb}^{2}(X) \backslash \operatorname{Hilb}^{2}(D / C)$. In fact, we can actually show that the connectedness of $M^{1}(v)$ by using Proposition 5.6. However, we omit the proof here.

In the following subsections, we will study the properties of these morphisms in details.

6.1. The diagram $\xi_{1}^{ \pm}$is a flip. In this subsection, we will show that the diagram $\xi_{1}^{ \pm}$is a flip. The main technical tool used here is so-called elementary transformation. First we observe the following.

Lemma 6.3. Let $Z \subset L_{y}$ be a length 2 closed subscheme $(y \in C)$. Then the following statements hold:

(1) We have an exact sequence

$$
0 \rightarrow I_{L_{y}} \rightarrow I_{Z} \rightarrow \mathcal{O}_{L_{y}}(-2) \rightarrow 0
$$

(2) We have $I_{L_{y}} \in \operatorname{Per}(X / Y)$.

In particular, the sequence (6.2) is the decomposition with respect to the torsion $\operatorname{pair}(\mathcal{T} \otimes \mathcal{O}(D), \mathcal{F} \otimes \mathcal{O}(D))$.

Proof. (1) Since $Z \subset L_{y}$ is length 2, we have

$$
0 \rightarrow \mathcal{O}_{L_{y}}(-2) \rightarrow \mathcal{O}_{L_{y}} \rightarrow \mathcal{O}_{Z} \rightarrow 0
$$


Hence we get the following commutative diagram:

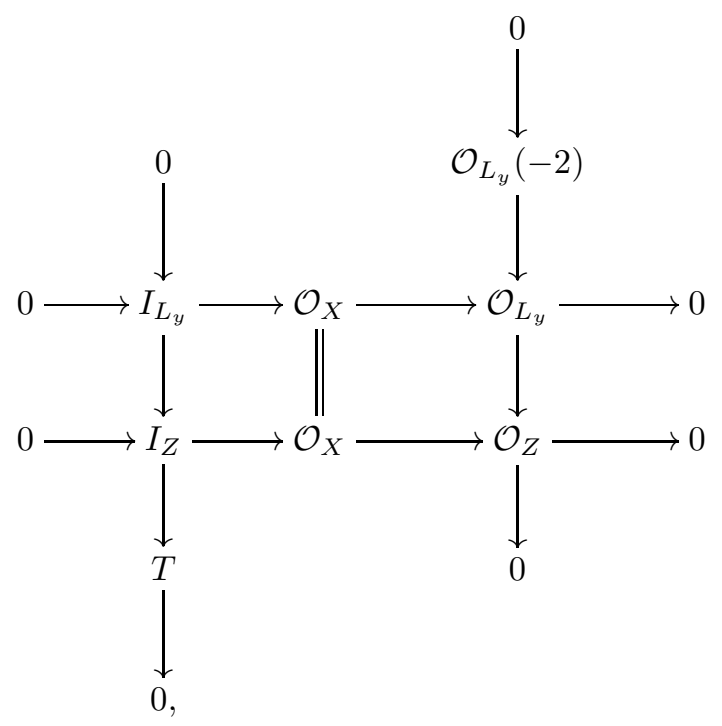

which implies $T=\mathcal{O}_{L_{y}}(-2)$ as required.

(2) Pulling back the exact sequence

$$
0 \rightarrow I_{y} \rightarrow \mathcal{O}_{Y} \rightarrow \mathcal{O}_{y} \rightarrow 0
$$

we get

$$
0 \rightarrow \mathcal{O}_{L_{y}}(-1) \rightarrow f^{*} I_{y} \rightarrow \mathcal{O}_{X} \rightarrow \mathcal{O}_{L_{y}} \rightarrow 0 .
$$

It gives two short exact sequences

$$
\begin{aligned}
& 0 \rightarrow \mathcal{O}_{L_{y}}(-1) \rightarrow f^{*} I_{y} \rightarrow I_{L_{y}} \rightarrow 0 \\
& 0 \rightarrow I_{L_{y}} \rightarrow \mathcal{O}_{X} \rightarrow \mathcal{O}_{L_{y}} \rightarrow 0 .
\end{aligned}
$$

By Lemma 4.1, we have $f^{*} I_{y} \in \operatorname{Per}(X / Y)$. Since there exists a surjection $f^{*} I_{y} \rightarrow I_{L_{y}}$, we also have $I_{L_{y}} \in \operatorname{Per}(X / Y)$ by Lemma 3.2 .

For the last assertion, we first note that $\mathcal{O}_{L_{y}}(-2) \otimes \mathcal{O}(-D) \cong \mathcal{O}_{L_{y}}(-1) \subset \mathcal{F}$. Furthermore, by (2) and Corollary 2.6. we have $I_{L_{y}}(-D) \in \mathcal{T}$. Hence the sequence (6.2) is the decomposition with respect to the torsion pair $(\mathcal{T} \otimes \mathcal{O}(D), \mathcal{F} \otimes \mathcal{O}(D))$.

Corollary 6.4. (1) The restriction of $\xi_{1}^{+}$to $\operatorname{Hilb}^{2}(D / C)$ is given by

$$
\xi_{1}^{+}: \operatorname{Hilb}^{2}(D / C) \rightarrow C, \quad I_{Z} \mapsto I_{L_{y}} .
$$

(2) For every closed point $y$ of $C$, the fiber of $\xi_{1}^{+}$over $I_{L_{y}}$ is given by

$$
\left(\xi_{1}^{+}\right)^{-1}\left(I_{L_{y}}\right)=\operatorname{Hilb}^{2}\left(L_{y}\right) \cong \mathbb{P}^{2} .
$$

(3) For every closed point $y$ of $C$, the fiber of $\xi_{1}^{-}$over $I_{L_{y}}$ is given by

$$
\left(\xi_{1}^{-}\right)^{-1}\left(I_{L_{y}}\right)=\mathbb{P E x t}^{1}\left(I_{L_{y}}, \mathcal{O}_{L_{y}}(-2)\right)^{\vee} \text {. }
$$

Proof. (1) Recall from Proposition 3.14 that the morphism $\xi_{1}^{+}$is defined by using the decomposition with respect to the torsion pair $(\mathcal{T} \otimes \mathcal{O}(D), \mathcal{F} \otimes \mathcal{O}(D))$. By Lemma 6.3 we have $\xi_{1}^{+}\left(I_{Z}\right)=I_{L_{y}}$ as required.

(2) By Corollary 6.2 and (1), for $I_{Z} \in \operatorname{Hilb}^{2}(X)$, we have $\xi_{1}^{+}\left(I_{Z}\right)=I_{L_{y}}$ if and only if $Z$ is the closed subscheme of $L_{y}$, i.e. $I_{Z} \in \operatorname{Hilb}^{2}\left(L_{y}\right)$. This proves the assertion. 
(3) For a 1-stable sheaf $E \in M^{1}(v)$, we have $E \in\left(\xi_{1}^{-}\right)^{-1}\left(I_{L_{y}}\right)$ if and only if there exists an element $T \in \mathcal{C}^{0} \otimes \mathcal{O}(D)$ such that $E$ fits into a non-trivial exact sequence

$$
0 \rightarrow T \rightarrow E \rightarrow I_{L_{y}} \rightarrow 0
$$

by the construction of the morphism $\xi_{1}^{-}$. Note that we have $\operatorname{ch}(T)=\operatorname{ch}(E)-$ $\operatorname{ch}\left(I_{L_{y}}\right)=\operatorname{ch}\left(I_{Z}\right)-\operatorname{ch}\left(I_{L_{y}}\right)=\operatorname{ch}\left(\mathcal{O}_{L_{y}}(-2)\right)$, where $I_{Z} \in \operatorname{Hilb}^{2}(X)$. Hence the only possibility is $T=\mathcal{O}_{L_{y}}(-2)$. We conclude that $\left(\xi_{1}^{-}\right)^{-1}\left(I_{L_{y}}\right)=\mathbb{P E x t}^{1}\left(I_{L_{y}}, \mathcal{O}_{L_{y}}(-2)\right)^{\vee}$.

The following lemma determines the dimension of $\mathbb{P} \operatorname{Ext}^{1}\left(I_{L_{y}}, \mathcal{O}_{L_{y}}(-2)\right)^{\vee}$ :

Lemma 6.5. For every $y \in C$, we have $\operatorname{ext}^{1}\left(I_{L_{y}}, \mathcal{O}_{L_{y}}(-2)\right)=n$.

Proof. Applying Hom $\left(-, \mathcal{O}_{L_{y}}(-2)\right)$ to the standard exact sequence

$$
0 \rightarrow I_{L_{y}} \rightarrow \mathcal{O}_{X} \rightarrow \mathcal{O}_{L_{y}} \rightarrow 0
$$

the claim is reduced to compute $\operatorname{ext}^{i}\left(\mathcal{O}_{L_{y}}, \mathcal{O}_{L_{y}}(-2)\right)$ for $i=1,2$. Using the localto-global spectral sequence

$$
E_{2}^{p, q}=H^{p}\left(X, \mathcal{E} x t^{q}\left(\mathcal{O}_{L_{y}}, \mathcal{O}_{L_{y}}(-2)\right)\right) \Rightarrow \operatorname{Ext}^{p+q}\left(\mathcal{O}_{L_{y}}, \mathcal{O}_{L_{y}}(-2)\right)
$$

and the isomorphism

$$
\begin{aligned}
\mathcal{E x t}^{q}\left(\mathcal{O}_{L_{y}}, \mathcal{O}_{L_{y}}(-2)\right) & \cong \bigwedge^{q} \mathcal{N}_{L_{y} / X} \otimes \mathcal{O}_{L_{y}}(-2) \\
& =\bigwedge_{q}^{q}\left(\mathcal{O}_{L_{y}}^{\oplus n-2} \oplus \mathcal{O}_{L_{y}}(-1)\right) \otimes \mathcal{O}_{L_{y}}(-2) \\
& \cong \mathcal{O}_{L_{y}}(-2)^{\oplus\left(\begin{array}{c}
n-2 \\
q
\end{array}\right)} \oplus \mathcal{O}_{L_{y}}(-3)^{\oplus\left(\begin{array}{c}
n-2 \\
q-1
\end{array}\right)}
\end{aligned}
$$

(cf. [8, Corollary 11.2]), we have

$$
\operatorname{ext}^{i}\left(\mathcal{O}_{L_{y}}, \mathcal{O}_{L_{y}}(-2)\right)= \begin{cases}1 & (i=1) \\ (n-2)+2=n & (i=2) .\end{cases}
$$

Using them, we get the result.

We next compute the normal bundle $\mathcal{N}_{\operatorname{Hilb}^{2}(D / C) / \operatorname{Hilb}^{2}(X)}$. To do that, let us define an embedding

$$
D \rightarrow C \times X, \quad x \mapsto(f(x), x)
$$

and denote the ideal sheaf of $D$ in $C \times X$ as $I_{D / C \times X}$. We also use the following notations for projections:
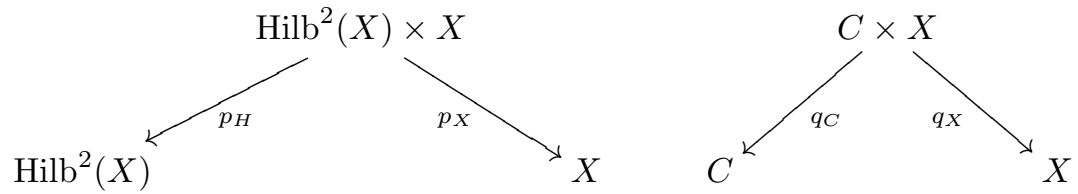

Definition 6.6. We define sheaves $\mathcal{E}_{ \pm} \in \operatorname{Coh}(C)$ and $\mathcal{E} \in \operatorname{Coh}\left(\operatorname{Hilb}^{2}(X)\right)$ as follows:

$$
\begin{aligned}
& \mathcal{E}_{+}:=\mathcal{E} x t_{q_{C}}^{1}\left(\mathcal{O}_{D}(2 D), I_{D / C \times X}\right), \quad \mathcal{E}_{-}:=\mathcal{E} x t_{q_{C}}^{1}\left(I_{D / C \times X}, \mathcal{O}_{D}(2 D)\right), \\
& \mathcal{E}:=\mathcal{E} x t_{p_{H}}^{1}(\mathcal{I}, \mathcal{I}) .
\end{aligned}
$$

We recall the following version of semicontinuity theorem: 
Theorem 6.7 (3, Satz 3]). Let $g: M \rightarrow N$ be a flat morphism between smooth projective varieties. Let $E, F \in \operatorname{Coh}(M)$ be flat sheaves over $N$. Fix an integer $i \in \mathbb{Z}_{\geq 0}$. Assume that for every $p \in N$, $\operatorname{ext}^{i}\left(E_{p}, F_{p}\right)$ is constant, where $E_{p}, F_{p} \in$ $\operatorname{Coh}\left(M_{p}\right)$ is the restriction of $E, F$ to the fiber $M_{p}:=g^{-1}(p)$. Then the sheaf $\mathcal{E} x t_{g}^{i}(E, F)$ is locally free. Furthermore, for $q=i-1, i, \mathcal{E} x t_{g}^{q}(E, F)$ commutes with the base change, i,e, for every $p \in N$, we have an isomorphism

$$
\left.\mathcal{E} x t_{g}^{q}(E, F)\right|_{\{p\}} \cong \operatorname{Ext}^{q}\left(E_{p}, F_{p}\right) .
$$

Corollary 6.8. (1) The sheaves $\mathcal{E}_{ \pm}, \mathcal{E}$ are locally free.

(2) We have $\operatorname{Hilb}^{2}(D / C) \cong \mathbb{P}\left(\mathcal{E}_{+}{ }^{\vee}\right)$.

(3) The Hilbert scheme $\operatorname{Hilb}^{2}(X)$ is smooth of dimension $2 n$ and its tangent bundle is given as $\mathcal{T}_{\mathrm{Hilb}^{2}(X)} \cong \mathcal{E}$.

Proof. First of all, the smoothness of $\operatorname{Hilb}^{2}(X)$ is well known: the Hilbert-Chow morphism $\operatorname{Hilb}^{2}(X) \rightarrow \operatorname{Sym}^{2}(X)$ coincides with $\mathrm{Bl}_{\Delta_{X}} \operatorname{Sym}^{2}(X)$, i.e. $\operatorname{Hilb}^{2}(X) \cong$ $\mathrm{Bl}_{\Delta_{X}} \operatorname{Sym}^{2}(X)$. Moreover, $\operatorname{Sym}^{2}(X)$ has only $\frac{1}{2}(1, \cdots, 1)$-type singularity along the diagonal $\Delta_{X} \subset \operatorname{Sym}^{2}(X)$. Hence $\operatorname{Hilb}^{2}(X)$ is smooth.

(1) Since $D$ is flat over $C, I_{D / C \times D}$ and $\mathcal{O}_{D}(2 D)$ are flat over $C$. Hence by Lemma 6.5 and Theorem 6.7 the assertion follows.

(2) We have the universal extension sheaf $\mathcal{F} \in \operatorname{Coh}\left(\mathbb{P}\left(\mathcal{E}_{+}^{\vee}\right) \times X\right)$ which fits into the exact sequence

$$
0 \rightarrow\left(\pi_{X}^{+}\right)^{*} I_{D / C \times X} \otimes p^{*} \mathcal{O}_{\pi^{+}}(1) \rightarrow \mathcal{F} \rightarrow\left(\pi_{X}^{+}\right)^{*} \mathcal{O}_{D}(2 D) \rightarrow 0
$$

(cf. 9, Example 2.1.12]). Here $\pi^{+}: \mathbb{P}\left(\mathcal{E}_{+}^{\vee}\right) \rightarrow C$ is the structure morphism of the projective space bundle, $\pi_{X}^{+}: \mathbb{P}\left(\mathcal{E}_{+}^{\vee}\right) \times X \rightarrow C \times X$ is the base change morphism, and $p: \mathbb{P}\left(\mathcal{E}_{+}^{\vee}\right) \times X \rightarrow \mathbb{P}\left(\mathcal{E}_{+}^{\vee}\right)$ is the projection. The sheaf $\mathcal{F}$ parametrizes all the extensions

$$
0 \rightarrow I_{L_{y}} \rightarrow F \rightarrow \mathcal{O}_{L_{y}}(-2) \rightarrow 0 .
$$

By Lemma 6.3, we have $F \cong I_{Z} \in \operatorname{Hilb}^{2}(D / C)$. Hence by the universality of the Hilbert scheme, we get $\operatorname{Hilb}^{2}(D / C) \cong \mathbb{P}\left(\mathcal{E}_{+}^{\vee}\right)$ as required.

(3) Consider the Kodaira-Spencer map $K S: \mathcal{T}_{\mathrm{Hilb}^{2}(X)} \rightarrow \mathcal{E}$. Since $\mathcal{E}$ commutes with the base change, $K S$ restricts to an isomorphism $K S_{p}: T_{p} \operatorname{Hilb}^{2}(X) \rightarrow$ $\operatorname{Ext}^{1}\left(I_{Z}, I_{Z}\right)$ for each $p=\left[I_{Z}\right] \in \operatorname{Hilb}^{2}(X)$. Hence $K S$ is surjective morphism between locally free sheaves of the same rank. We conclude that $\mathcal{T}_{\text {Hilb }^{2}(X)} \cong \mathcal{E}$.

Now we can compute the normal bundle:

Lemma 6.9 (cf. [5, Proposition 3.7]). We have an isomorphism

$$
\mathcal{N}_{\mathrm{Hilb}^{2}(D / C) / \mathrm{Hilb}^{2}(X)} \cong\left(\pi^{+}\right)^{*} \mathcal{E}_{-} \otimes \mathcal{O}_{\pi^{+}}(-1) .
$$

Proof. First we construct a morphism $\left.\mathcal{E}\right|_{\mathrm{Hilb}^{2}(D / C)} \rightarrow\left(\pi^{+}\right)^{*} \mathcal{E}_{-} \otimes \mathcal{O}_{\pi^{+}}(-1)$. Applying $\mathbf{R} \pi_{*}^{+} \mathbf{R} \mathcal{H o m}\left(\left.\mathcal{I}\right|_{\mathrm{Hibb}^{2}(D / C)},-\right)$ and $\mathbf{R} \pi_{*}^{+} \mathbf{R} \mathcal{H}$ om $\left(-,\left(\pi_{X}^{+}\right)^{*} \mathcal{O}_{D}(2 D)\right)$ to the exact sequence (6.3) and taking its cohomology, we get

$$
\delta_{1}:\left.\mathcal{E}\right|_{\mathrm{Hilb}^{2}(D / C)} \rightarrow \mathcal{E} x t_{\pi^{+}}^{1}\left(\left.\mathcal{I}\right|_{\mathrm{Hilb}^{2}(D / C)},\left(\pi_{X}^{+}\right)^{*} \mathcal{O}_{D}(2 D)\right)
$$

$$
\begin{aligned}
\delta_{2}: \mathcal{E} x t_{\pi^{+}}^{1} & \left(\left.\mathcal{I}\right|_{\mathrm{Hilb}^{2}(D / C)},\left(\pi_{X}^{+}\right)^{*} \mathcal{O}_{D}(2 D)\right) \rightarrow \\
& \mathcal{E} x t_{\pi^{+}}^{1}\left(\left(\pi_{X}^{+}\right)^{*} I_{D / C \times X} \otimes p^{*} \mathcal{O}_{\pi^{+}}(1),\left(\pi_{X}^{+}\right)^{*} \mathcal{O}_{D}(2 D)\right) .
\end{aligned}
$$

Note that we used $\left.\mathcal{F} \cong \mathcal{I}\right|_{\operatorname{Hilb}^{2}(D / C)}$ above. Straightforward computation shows that

$$
\mathcal{E} x t_{\pi^{+}}^{1}\left(\left(\pi_{X}^{+}\right)^{*} I_{D / C \times X} \otimes p^{*} \mathcal{O}_{\pi^{+}}(1),\left(\pi_{X}^{+}\right)^{*} \mathcal{O}_{D}(2 D)\right) \cong\left(\pi^{+}\right)^{*} \mathcal{E}^{-} \otimes \mathcal{O}_{\pi^{+}}(-1) .
$$


Hence we get the morphism

$$
\delta:=\delta_{2} \circ \delta_{1}:\left.\mathcal{E}\right|_{\operatorname{Hilb}^{2}(D / C)} \rightarrow\left(\pi^{+}\right)^{*} \mathcal{E}_{-} \otimes \mathcal{O}_{\pi^{+}}(-1) .
$$

We will prove that $\delta$ is surjective and $\operatorname{ker} \delta \cong \mathcal{T}_{\mathrm{Hilb}^{2}(D / C)}$. To show that, it is enough to show the following: For every $p=\left[I_{Z}\right] \in \operatorname{Hilb}^{2}(D / C)$ with $Z \subset L_{y}$,

(1) the restriction $\delta_{p}:\left.T_{p} \operatorname{Hilb}^{2}(X)\right|_{\operatorname{Hilb}^{2}(D / C)} \rightarrow \operatorname{Ext}^{1}\left(I_{L_{y}}, \mathcal{O}_{L_{y}}(-2)\right)$ is surjective,

(2) $\operatorname{ker}\left(\delta_{p}\right)=T_{p} \operatorname{Hilb}^{2}(D / C)$.

In (1), we can actually show that both $\delta_{1, p}$ and $\delta_{2, p}$ are surjective by using the spectral sequence argument as in Lemma 6.5.

For (2), it is now enough to show that $T_{p} \operatorname{Hilb}^{2}(D / C) \subset \operatorname{ker}\left(\delta_{p}\right)$ since they are vector spaces of the same dimension. The argument is exactly same as [5. Proposition 3.7] and hence we omit the detail.

The following result directly follows from Lemma 6.9.

Corollary 6.10. (1) When $n=2, \xi_{1}^{+}$is a flipping contraction.

(2) When $n=3, \xi_{1}^{+}$is a flopping contraction.

(3) When $n \geq 4, \xi_{1}^{+}$is an anti-flipping contraction.

Let $\mu: M:=\mathrm{Bl}_{\operatorname{Hilb}^{2}(D / C)} \operatorname{Hilb}^{2}(X) \rightarrow \operatorname{Hilb}^{2}(X), E \subset M$ be the $\mu$-exceptional divisor, $\nu:=\left.\mu\right|_{E}: D \rightarrow \operatorname{Hilb}^{2}(D / C)$. Note that by Lemma 6.9, we know that $E \cong \mathbb{P}\left(\mathcal{E}_{-}^{\vee}\right) \times_{C} \mathbb{P}\left(\mathcal{E}_{+}^{\vee}\right)$. By the elementary transformation, we will construct the family $\mathcal{G}$ of 1 -stable sheaves on $M$, which gives us the morphism $M \rightarrow \widetilde{M}^{1}(v)$.

Pulling back the exact sequence (6.3), we get

$$
\left.0 \rightarrow \nu_{X}^{*}\left(\pi_{X}^{+}\right)^{*}\left(I_{D / C \times X} \otimes\left(\mathcal{O}_{\pi^{+}}(1)\right)\right) \rightarrow \mu_{X}^{*} \mathcal{I}\right|_{E \times X} \rightarrow \nu_{X}^{*}\left(\pi^{+}\right)^{*} \mathcal{O}_{D}(2 D) \rightarrow 0
$$

in $\operatorname{Coh}(M \times X)$. Now define

$$
\mathcal{G}:=\operatorname{ker}\left(\left.\mu_{X}^{*} \mathcal{I} \rightarrow \mu_{X}^{*} \mathcal{I}\right|_{E \times X} \rightarrow \nu_{X}^{*}\left(\pi^{+}\right)^{*} \mathcal{O}_{D}(2 D)\right) .
$$

Lemma 6.11. (1) The sheaf $\mathcal{G}$ is a flat family of 1-stable sheaves and hence defines the morphism $\sigma: M \rightarrow \widetilde{M}^{1}(v)$.

(2) The restriction $\left.\sigma\right|_{E}: E \rightarrow \sigma(E)$ coincides with the projection morphism $\mathbb{P}\left(\mathcal{E}_{-}^{\vee}\right) \times_{C} \mathbb{P}\left(\mathcal{E}_{+}^{\vee}\right) \rightarrow \mathbb{P}\left(\mathcal{E}_{-}^{\vee}\right)$.

Proof. (1) We get the following commutative diagram:

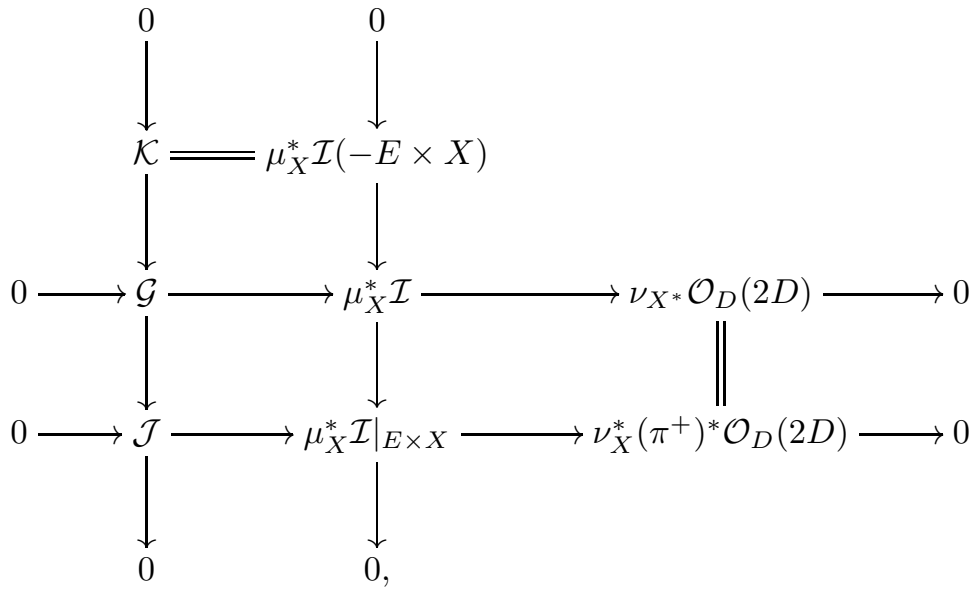

where we put

$$
\mathcal{J}:=\nu_{X}^{*}\left(\pi_{X}^{+}\right)^{*}\left(I_{D / C \times X} \otimes \mathcal{O}_{\pi^{+}}(1)\right)
$$


Applying $(-) \otimes^{\mathbf{L}} \mathcal{O}_{E \times X}$ to the left column in the above diagram, we get the following long exact sequence:

$$
\begin{aligned}
0 \rightarrow & \mathcal{T}_{\text {or }^{1}}\left(\mathcal{O}_{E \times X}, \mathcal{G}\right) \rightarrow \mathcal{T}_{\text {or }^{1}}\left(\mathcal{O}_{E \times X}, \mathcal{J}\right) \\
& \left.\left.\mu_{X}^{*} \mathcal{I}(-E \times X)\right|_{E \times X} \rightarrow \mathcal{G}\right|_{E \times X} \rightarrow \mathcal{J} \rightarrow 0
\end{aligned}
$$

Note that we have $\mathcal{T}$ or ${ }^{1}\left(\mathcal{O}_{E \times X}, \mathcal{K}\right)=0$ since $\mathcal{I}$ is flat over $\operatorname{Hilb}^{2}(X)$. Using the isomorphism $\mathcal{O}_{E \times X} \cong\left(\mathcal{O}_{M \times X}(-E \times X) \rightarrow \mathcal{O}_{M \times X}\right)$ in $D^{b}(M \times X)$, we can easily show that $\mathcal{T}_{o r}{ }^{1}\left(\mathcal{O}_{E \times X}, \mathcal{G}\right)=0$ and the above long exact sequence splits into

$$
\begin{aligned}
& \left.0 \rightarrow \mathcal{J}(-E \times X) \rightarrow \mu_{X}^{*} \mathcal{I}(-E \times X)\right|_{E \times X} \rightarrow\left(\nu_{X}^{*}\left(\pi^{+}\right)^{*} \mathcal{O}_{D}(2 D)\right)(-E \times X) \rightarrow 0 \\
& \left.0 \rightarrow\left(\nu_{X}^{*}\left(\pi^{+}\right)^{*} \mathcal{O}_{D}(2 D)\right)(-E \times X) \rightarrow \mathcal{G}\right|_{E \times X} \rightarrow \mathcal{J} \rightarrow 0 .
\end{aligned}
$$

Hence $\mathcal{G}$ is flat over $M$. Furthermore, $\left.\mathcal{G}\right|_{E \times X}$ parametrizes extensions

$$
0 \rightarrow \mathcal{O}_{L_{y}}(-2) \rightarrow G \rightarrow I_{L_{y}} \rightarrow 0
$$

i.e. 1-stable objects $G$. This defines a morphism $\sigma: M \rightarrow M^{1}(v)$. We claim that the image of $\sigma$ is contained in a connected component $\widetilde{M}^{1}(v)$. Indeed, we have $\sigma(M \backslash E) \subset \widetilde{M}^{1}(v)$ by our definition of $\widetilde{M}^{1}(v)$. Since $\sigma(M)$ is connected, it must be contained in $\widetilde{M}^{1}(v)$.

(2) The assertion follows from [4, Proposition A.2].

Corollary 6.12. The scheme $\widetilde{M}^{1}(v)$ is a smooth projective variety. Moreover,

(1) When $n=2, \operatorname{Hilb}^{2}(X) \rightarrow \widetilde{M}^{1}(v)$ is a flip.

(2) When $n=3$, $\operatorname{Hilb}^{2}(X) \rightarrow \widetilde{M}^{1}(v)$ is a flop.

(3) When $n \geq 4$, $\operatorname{Hilb}^{2}(X) \rightarrow \widetilde{M}^{1}(v)$ is an anti-flip.

Proof. Note that Lemma 6.11 shows that the diagram

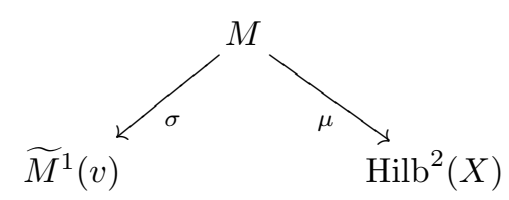

is a family version of the standard flip (cf. [8, page 258]). Since we already know the projectivity of $\widetilde{M}^{1}(v)$, it remains to check that $\widetilde{M}^{1}(v)$ is a smooth variety. The assertion then follows from the Fujiki-Nakano criterion (cf. [6] and [8, page 259]).

6.2. $\xi_{0}$ is an extremal contraction. In this subsection, we describe the fiber of $\xi_{0}$ and show that it is the contraction of a $K$-negative extremal ray. Recall from Corollary 6.4 (3) that we have

$$
\widetilde{M}^{1}(v)=\left(\operatorname{Hilb}^{2}(X) \backslash \operatorname{Hilb}^{2}(D / C)\right) \coprod \mathbb{P}\left(\mathcal{E}_{-}^{\vee}\right)
$$

We start with the following lemma:

Lemma 6.13. Let $G \in \mathbb{P}\left(\mathcal{E}_{-}^{\vee}\right) \subset \widetilde{M}^{1}(v)$. Then $G$ is not 0 -stable.

Proof. We have an exact sequence

$$
0 \rightarrow \mathcal{O}_{L_{y}}(-2) \rightarrow G \rightarrow I_{L_{y}} \rightarrow 0
$$

for some $y \in C$. Applying $\operatorname{Hom}\left(-, \mathcal{O}_{L_{y}}(-1)\right)$, we get

$$
\begin{aligned}
& \operatorname{Hom}\left(I_{L_{y}}, \mathcal{O}_{L_{y}}(-1)\right) \rightarrow \operatorname{Hom}\left(G, \mathcal{O}_{L_{y}}(-1)\right) \rightarrow \operatorname{Hom}\left(\mathcal{O}_{L_{y}}(-2), \mathcal{O}_{L_{y}}(-1)\right) \\
\rightarrow & \operatorname{Ext}^{1}\left(I_{L_{y}}, \mathcal{O}_{L_{y}}(-1)\right)
\end{aligned}
$$


Since $I_{L_{y}} \in \operatorname{Per}(X / Y)$, Hom $\left(I_{L_{y}}, \mathcal{O}_{L_{y}}(-1)\right)=0$. Moreover, as in Lemma 6.5. we can show that $\operatorname{ext}^{1}\left(I_{L_{y}}, \mathcal{O}_{L_{y}}(-1)\right)=1$. Hence hom $\left(G, \mathcal{O}_{L_{y}}(-1)\right)=1 \neq 0$. This shows that $G \notin \operatorname{Per}(X / Y)$.

By the above lemma and Lemma 6.1 the exceptional locus of $\xi_{0}$ is

$$
\operatorname{Exc}\left(\xi_{0}\right)=\left(\left\{I_{Z} \in \operatorname{Hilb}^{2}(X): Z \cap D \neq \emptyset\right\} \backslash \operatorname{Hilb}^{2}(D / C)\right) \coprod \mathbb{P}\left(\mathcal{E}_{-}^{\vee}\right) .
$$

To study the geometry of $\xi_{0}$, we introduce the following filtration $B_{1} \subset B_{2} \subset$ $B_{3}=B \subset \operatorname{Hilb}^{2}(Y)$ of $\operatorname{Hilb}^{2}(Y)$ :

- $B_{1}:=\left\{I_{W} \in \operatorname{Hilb}^{2}(C): \operatorname{Supp}\left(I_{W}\right)=\mathrm{pt}\right\}$,

- $B_{2}:=\operatorname{Hilb}^{2}(C)$,

- $B_{3}:=\left\{I_{W} \in \operatorname{Hilb}^{2}(Y): W \cap C \neq \emptyset\right\}$.

Lemma 6.14. (1) We have $\xi_{0}\left(\operatorname{Exc}\left(\xi_{0}\right)\right)=B$.

(2) For $I_{W} \in B \backslash B_{2}$, the fibre of $\xi_{0}$ is $\xi_{0}^{-1}\left(I_{W}\right)=\mathbb{P}^{1}$.

(3) For $I_{W} \in B_{2} \backslash B_{1}$, the fibre of $\xi_{0}$ is $\xi_{0}^{-1}\left(I_{W}\right)=\mathbb{P}^{1} \times \mathbb{P}^{1}$.

(4) For $I_{W} \in B_{1}$, the fibre of $\xi_{0}$ is the weighted projective plane $\xi_{0}^{-1}\left(I_{W}\right)=$ $\mathbb{P}(1,1,2)$.

Here, we consider the image and the fibers of $\xi_{0}$ with its reduced scheme structures.

Proof. (1) First let $I_{Z} \in \operatorname{Exc}\left(\xi_{0}\right) \cap \operatorname{Hilb}^{2}(X)$. Then pushing forward the exact sequence

$$
0 \rightarrow I_{Z} \rightarrow \mathcal{O}_{X} \rightarrow \mathcal{O}_{Z} \rightarrow 0
$$

we get

$$
0 \rightarrow f_{*} I_{Z} \rightarrow \mathcal{O}_{Y} \rightarrow f_{*} \mathcal{O}_{Z} \rightarrow 0 .
$$

Hence $f_{*} I_{Z}$ is an ideal sheaf of length 2 closed subscheme $W$ of $\mathrm{Y}$ with Supp $W=$ $f(Z)$. Since $Z \cap D \neq \emptyset, W \cap C \neq \emptyset$, i.e. $f_{*} I_{Z} \in B$.

Next take $G \in \mathbb{P}\left(\mathcal{E}_{-}^{\vee}\right)$. We can easily check that the following natural map $\alpha: \operatorname{Ext}^{1}\left(I_{L_{y}}, \mathcal{O}_{L_{y}}(-2)\right) \rightarrow \operatorname{Ext}^{1}\left(\mathcal{O}_{y}, \mathcal{O}_{y}\right)$ determines the class of $f_{*} G \in \operatorname{Hilb}^{2}(Y)$ : Consider the short exact sequence

$$
0 \rightarrow \mathcal{O}_{L_{y}}(-1) \rightarrow f^{*} I_{y} \rightarrow I_{L_{y}} \rightarrow 0
$$

Applying $\operatorname{Hom}\left(-, \mathcal{O}_{L_{y}}(-2)\right)$, we get

$$
\begin{aligned}
\alpha: \operatorname{Ext}^{1}\left(I_{L_{y}}, \mathcal{O}_{L_{y}}(-2)\right) & \rightarrow \operatorname{Ext}^{1}\left(f^{*} I_{y}, \mathcal{O}_{L_{y}}(-2)\right) \\
& \cong \operatorname{Hom}\left(I_{y}, \mathbf{R} f_{*} \mathcal{O}_{L_{y}}(-2)[1]\right) \\
& \cong \operatorname{Hom}\left(I_{y}, \mathcal{O}_{y}\right) \\
& \cong \operatorname{Ext}^{1}\left(\mathcal{O}_{y}, \mathcal{O}_{y}\right) .
\end{aligned}
$$

Hence $f_{*} G \in B_{1}$. Furthermore, we have $\operatorname{Ext}^{1}\left(\mathcal{O}_{L_{y}}(-1), \mathcal{O}_{L_{y}}(-2)\right)=0$ as in Lemma 6.5 and hence $\alpha$ is injective. Since we know $\operatorname{ext}^{1}\left(I_{L_{y}}, \mathcal{O}_{L_{y}}(-2)\right)=$ $\operatorname{ext}^{1}\left(\mathcal{O}_{y}, \mathcal{O}_{y}\right)=n, \alpha$ is bijective. We conclude that

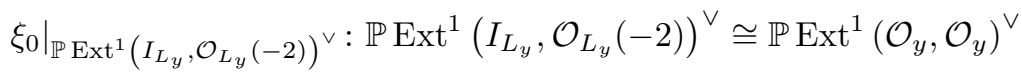

is an isomorphism.

(2) Take an element $I_{W} \in B \backslash B_{2}$. First assume that $W=\{a, b\}, a \in C, b \notin C$. Then from the argument of (1), we have

$$
\xi_{0}^{-1}\left(I_{W}\right)=\left\{I_{p, q} \in \operatorname{Hilb}^{2}(X): f(p)=a, f(q)=b\right\} \cong L_{a}
$$


Next assume that $\operatorname{Supp} W=\{y\}$, but scheme-theoretically $W \nsubseteq C$. Let $x \in D$ with $f(x)=y$. Then the following commutative diagram of the tangent maps determines the morphism

$$
\xi_{0}: \mathbb{P E x t}_{X}^{1}\left(\mathcal{O}_{x}, \mathcal{O}_{x}\right)^{\vee} \backslash \mathbb{P} \operatorname{Ext}_{L_{y}}^{1}\left(\mathcal{O}_{x}, \mathcal{O}_{x}\right)^{\vee} \rightarrow \mathbb{P E x t}_{Y}^{1}\left(\mathcal{O}_{y}, \mathcal{O}_{y}\right)^{\vee} .
$$

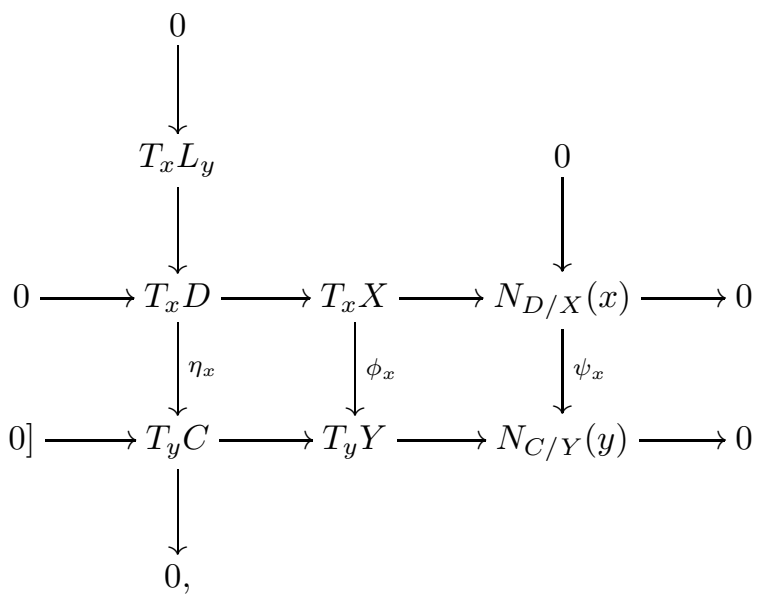

where maps $\eta_{x}, \phi_{x}, \psi_{x}$ are the tangent maps. Note that the point $\left[\psi_{x}\left(N_{D / X}(x)\right)^{\vee}\right] \in$ $\mathbb{P}\left(N_{C / Y}(y)^{\vee}\right)=L_{y}$ is nothing but $x \in L_{y}$. Now take the 1-dimensional subspace $\mathbb{C} \cdot(\alpha, \beta) \subset T_{y} Y=T_{y} C \oplus N_{C / Y}(y)$ which corresponds to $I_{W}$. Since we assume that $W \nsubseteq C$, we have $0 \neq \beta \in N_{C / Y}(y)$. Let $x:=\left[\left(\mathbb{C} \cdot \beta^{\vee}\right)\right] \in L_{y}$. Then we have $\phi_{x}^{-1}(\mathbb{C} \cdot(\alpha, \beta))=T_{x} L_{y} \oplus N_{D / X}(x)$. Moreover, recall from (1) that we have the unique element $G_{W} \in \mathbb{P E x t}^{1}\left(I_{L_{y}}, \mathcal{O}_{L_{y}}(-2)\right)^{\vee}$ such that $f_{*} G_{W}=I_{W}$. Hence we conclude that

$$
\begin{aligned}
\xi_{0}^{-1}\left(I_{W}\right) & =\left(\mathbb{P}\left(T_{x} L_{y} \oplus N_{D / X}(x)\right)^{\vee} \backslash \mathbb{P}\left(T_{x} L_{y}\right)^{\vee}\right) \coprod\left\{G_{W}\right\} \\
& =\mathbb{A}^{1} \coprod \mathrm{pt} .
\end{aligned}
$$

Since both $\widetilde{M}^{1}(v)$ and $\operatorname{Hilb}^{2}(Y)$ are smooth, $H^{1}\left(\mathcal{O}_{\xi_{0}^{-1}\left(I_{W}\right)}\right)=0$. Hence we must have $\xi_{0}^{-1}\left(I_{W}\right) \cong \mathbb{P}^{1}$.

(3) Take an element $I_{W} \in B_{2} \backslash B_{1}$. Then by definition, $W=\{a, b\}$ with $a, b \in C$, $a \neq b$. Hence we have

$$
\xi_{0}^{-1}\left(I_{W}\right)=\left\{I_{p, q}: f(p)=a, f(q)=b\right\}=L_{a} \times L_{b} \cong \mathbb{P}^{1} \times \mathbb{P}^{1} .
$$

(4) Let $I_{W} \in B_{1}$, Supp $W=\{y\} \subset C$. Take the subspace $\mathbb{C} \cdot \alpha \subset T_{y} Y$ corresponding to $I_{W}$. Since $W \subset C$ as scheme, we have $\mathbb{C} \cdot \alpha \subset T_{y} C$. For each $x \in L_{y}$, we have $\phi_{x}^{-1}(\mathbb{C} \cdot \alpha)=\mathbb{C} \cdot \alpha \oplus T_{x} L_{y}$. If we change $x \in L_{y}$, the vector space $T_{x} L_{y}$ changes, but the subspace $\mathbb{C} \cdot \alpha$ does not change. As before, we also have the unique element $G_{W} \in \mathbb{P} \operatorname{Ext}^{1}\left(I_{L_{y}}, \mathcal{O}_{L_{y}}(-2)\right)^{\vee}$ such that $f_{*} G_{W}=I_{W}$. Hence we conclude that

$$
\begin{aligned}
\xi_{0}^{-1}\left(I_{W}\right) & =\left(\mathbb{P}\left(\left(\mathcal{O}_{L_{y}} \oplus \mathcal{T}_{L_{y}}\right)^{\vee}\right) \backslash \mathbb{P}\left(\mathcal{T}_{L_{y}}^{\vee}\right)\right) \coprod\left\{G_{W}\right\} \\
& \cong\left(\mathbb{P}\left(\mathcal{O}_{L_{y}} \oplus \mathcal{O}_{L_{y}}(-2)\right) \backslash \mathbb{P}\left(\mathcal{O}_{L_{y}}(-2)\right)\right) \coprod\left\{G_{W}\right\}
\end{aligned}
$$

By this description, we can see that $\xi_{0}^{-1}\left(I_{W}\right)$ is the proper transform of $S:=$ $\mathbb{P}\left(\mathcal{O}_{L_{y}} \oplus \mathcal{O}_{L_{y}}(-2)\right)$ via the birational map $\widetilde{M}^{1}(v) \rightarrow \operatorname{Hilb}^{2}(X)$. To determine the 
scheme structure of $\xi_{0}^{-1}\left(I_{W}\right)$, recall the diagram

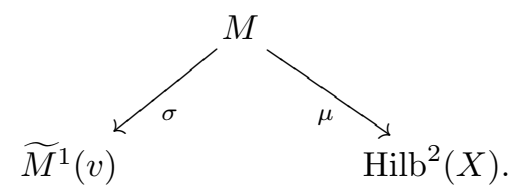

Since $S \cap \operatorname{Hilb}^{2}(D / C)=\mathbb{P}\left(\mathcal{O}_{L_{y}}(-2)\right)=: b$, the proper transform of $S$ by $\mu$ is $\mu_{*}^{-1}(S) \cong S$. Then the morphism $\left.\sigma\right|_{S}: S \cong \mu_{*}^{-1}(S) \rightarrow \xi_{0}^{-1}\left(I_{W}\right)$ is nothing but the contraction of a $(-2)$-curve $b \subset S$ and hence we get $\xi_{0}^{-1}\left(I_{W}\right) \cong \mathbb{P}(1,, 1,2)$ as required.

Lemma 6.15. The relative Picard number $\rho\left(\widetilde{M}^{1}(v) / \operatorname{Hilb}^{2}(Y)\right)$ is one.

Proof. By Lemma 6.14, the only codimension 1 irreducible component in $\operatorname{Exc}\left(\xi_{0}\right)$ is the closure of $\xi_{0}^{-1}\left(B \backslash B_{2}\right)$. Hence it is enough to show that $B$ is irreducible. To see that, we may assume $Y=\mathbb{C}^{n}=\operatorname{Spec} \mathbb{C}\left[x_{1}, \cdots, x_{n}\right], C=\left(x_{1}=x_{2}=0\right)$. Then we can write down the local equations of $B \subset \operatorname{Hilb}^{2}(Y)$ by using the fact that $\operatorname{Hilb}^{2}(Y)=\mathrm{Bl}_{\Delta_{Y}} \operatorname{Sym}^{2}(Y)$. The Jacobian criterion then shows that $B$ is smooth. In particular, it is irreducible.

Corollary 6.16. The birational morphism $\xi_{0}$ is the contraction of a $K$-negative extremal ray.

Proof. By Lemma 6.15, we know that $\xi_{0}$ is the contraction of an extremal ray $R$. Hence it is enough to compute the intersection number $K_{\widetilde{M}^{1}(v)} \cdot f$ for one element $f \in R$. Fix $y \in C, q \in X \backslash D$ and put $f:=\left\{I_{p, q}: p \in L_{y}\right\} \in R$. Since $f$ does not intersects with the exceptional divisor of the Hilbert-Chow morphism $\operatorname{Hilb}^{2}(X) \rightarrow \operatorname{Sym}^{2}(X)$, we have

$$
K_{\widetilde{M}^{1}(v)} \cdot f=K_{\mathrm{Hilb}^{2}(X)} \cdot f=K_{X \times X} \cdot\left(L_{y} \times\{q\}\right)=-1 .
$$

Remark 6.17. When $n=2$, the locus $\operatorname{Hilb}^{2}(C)=\emptyset$. Hence all the fibers of $\xi_{0}$ are $\mathbb{P}^{1}$. In general, Nakajima and Yoshioka shows that every fibre of the zig-zag diagram (1.1) is the Grassmann variety (see Theorem 1.2).

On the other hand, for $n \geq 3$, we have shown that $\mathbb{P}^{1} \times \mathbb{P}^{1}$ and $\mathbb{P}(1,1,2)$ appear as the fibers of $\xi_{0}$. Of course, they are not Grassmann variety. Furthermore, $\mathbb{P}(1,1,2)$ is even singular. This shows that for $n \geq 3$, more complicated fibers appear in the zig-zag diagram.

Acknowledgement. I would like to thank my supervisor Professor Yukinobu Toda, who gave me various advices and comments. I would also like to thank Takeru Fukuoka, Yousuke Matsuzawa, Masaru Nagaoka, and Genki Ouchi for fruitful discussions, and Wahei Hara, Yuki Hirano for reading the draft version of this article and giving useful comments.

This work was supported by the program for Leading Graduate Schools, the Ministry of Education,Culture,Sports,Science and Technology, Japan; and Grantin-Aid for Japan Society for the Promotion of Science Research Fellow [17J00664].

\section{REFERENCES}

[1] A. Bodzenta and A. Bondal. Canonical tilting relative generators. Adv. Math., 323:226-278, 2018.

[2] T. Bridgeland. Flops and derived categories. Invent. Math., 147(3):613-632, 2002.

[3] C. Bănică, M. Putinar, and G. Schumacher. Variation der globalen Ext in Deformationen kompakter komplexer Räume. Math. Ann., 250(2):135-155, 1980. 
[4] R. Friedman. Vector bundles and SO(3)-invariants for elliptic surfaces. J. Amer. Math. Soc., 8(1):29-139, 1995.

[5] R. Friedman and Z. Qin. Flips of moduli spaces and transition formulas for Donaldson polynomial invariants of rational surfaces. Comm. Anal. Geom., 3(1-2):11-83, 1995.

[6] A. Fujiki and S. Nakano. Supplement to "On the inverse of monoidal transformation". Publ. Res. Inst. Math. Sci., 7:637-644, 1971/72.

[7] D. Happel, I. Reiten, and S. O. Smalø. Tilting in abelian categories and quasitilted algebras. Mem. Amer. Math. Soc., 120(575):viii+ 88, 1996.

[8] D. Huybrechts. Fourier-Mukai transforms in algebraic geometry. Oxford Mathematical Monographs. The Clarendon Press, Oxford University Press, Oxford, 2006.

[9] D. Huybrechts and M. Lehn. The geometry of moduli spaces of sheaves. Aspects of Mathematics, E31. Friedr. Vieweg \& Sohn, Braunschweig, 1997.

[10] A. Langer. Semistable sheaves in positive characteristic. Ann. of Math. (2), 159(1):251-276, 2004.

[11] K. Matsuki and R. Wentworth. Mumford-Thaddeus principle on the moduli space of vector bundles on an algebraic surface. Internat. J. Math., 8(1):97-148, 1997.

[12] H. Nakajima and K. Yoshioka. Perverse coherent sheaves on blow-up. I. A quiver description. In Exploring new structures and natural constructions in mathematical physics, volume 61 of Adv. Stud. Pure Math., pages 349-386. Math. Soc. Japan, Tokyo, 2011.

[13] H. Nakajima and K. Yoshioka. Perverse coherent sheaves on blow-up. II. Wall-crossing and Betti numbers formula. J. Algebraic Geom., 20(1):47-100, 2011.

[14] H. Nakajima and K. Yoshioka. Perverse coherent sheaves on blowup, III: Blow-up formula from wall-crossing. Kyoto J. Math., 51(2):263-335, 2011.

[15] D. O. Orlov. Projective bundles, monoidal transformations, and derived categories of coherent sheaves. Izv. Ross. Akad. Nauk Ser. Mat., 56(4):852-862, 1992.

[16] A. Schmitt. Walls for Gieseker semistability and the Mumford-Thaddeus principle for moduli spaces of sheaves over higher dimensional bases. Comment. Math. Helv., 75(2):216-231, 2000.

[17] Y. Toda. Curve counting theories via stable objects II: DT/ncDT flop formula. J. Reine Angew. Math., 675:1-51, 2013.

[18] Y. Toda. Stability conditions and extremal contractions. Math. Ann., 357(2):631-685, 2013.

[19] Y. Toda. Stability conditions and birational geometry of projective surfaces. Compos. Math., 150(10):1755-1788, 2014.

[20] M. Van den Bergh. Three-dimensional flops and noncommutative rings. Duke Math. J., 122(3):423-455, 2004. 